LPTHE Orsay 93/72

cond-mat/9507092

\title{
Theory of the critical state of low-dimensional spin glass
}

\author{
M.J. Thill and H.J. Hilhorst \\ Laboratoire de Physique Théorique et Hautes Energies (CNRS - URA 63), \\ Bâtiment 211, Université de Paris-Sud, F-91405 Orsay CEDEx
}

(11th May 2018)

\begin{abstract}
We analyse the critical region of finite- $(d)$-dimensional Ising spin glass, in particular the limit of $d$ closely above the lower critical dimension $d_{\ell}$. At criticality the thermally active degrees of freedom are surfaces (of width zero) enclosing clusters of spins that may reverse with respect to their environment. The surfaces are organised in finite interacting structures. These may be called protodroplets, since in the off-critical limit they reduce to the Fisher and Huse droplets. This picture provides an explanation for the phenomenon of critical chaos discovered earlier. It also implies that the spin-spin and energy-energy correlation functions are multifractal and we present scaling laws that describe them. Several of our results should be verifiable in Monte Carlo studies at finite temperature in $d=3$.

PACS numbers: 05.50.+q, 64.60.Fr, 75.50.Lk
\end{abstract}

\section{INTRODUCTION}

No exact solution exists of the spin glass problem in the physical dimensions $d=2$ and $d=3$. The most successful approximate description was developed by McMillan [1], Bray and Moore [2], and Fisher and Huse [3, 14. The authors [2] speak of a scaling theory and the authors [3, 4] of a droplet theory. In both cases the main hypothesis is the existence of an effective coupling constant $K^{L}$ dependent on the length scale $L$ and satisfying scaling laws near zero temperature (with thermal exponent $y$ ) and at criticality (with exponent $y_{c}$ ). These scaling laws may also be thought of as arising from a renormalisation group ( $R G$ ) for the spin glass, and in fact real space $R G$ arguments are used by all the authors [1]. In particular, RG arguments can be put forward [glass is well described by the Edwards-Anderson (EA) Ising spin glass model [6].

Again, no exact RG is known for the finite-dimensional spin glass in dimensions $d>1$. The most popular approximate RG is the Migdal-Kadanoff (MK) construction [7 10. In spite of its evident shortcomings the MKRG has been successful as an approximate calculation scheme for ferromagnets and other nonrandom systems. For the finite-dimensional spin glass there does not today exist a better alternative.

Upon comparison one finds that the MKRG results for the spin glass are close, if not identical, to those obtained by the scaling and droplet theory. Most conclusions from MKRG are easily interpreted in the language of the droplet theory and the differences where they appear can be easily understood. There is therefore no reason at this level of approximation not to work with the MKRG, or, for that matter, with any real space RG of the same ilk. In this work we shall exploit the potential of the MKRG for spin glasses more fully than has been done hitherto. Our aim is to explain the phenomenon of "chaos" known to occur at criticality in low enough dimensions 11, 12. We achieve this goal by our 
interpretation of new MKRG results. We are led, in particular, to a characterisation of the thermally active degrees of freedom in critical Ising spin glass just above their lower critical dimension.

In order to set the stage for our work, we recall the most essential ones of the established results on zero temperature and critical chaos.

A droplet of linear size $L$ at a point $\boldsymbol{R}$ in space is defined as the domain of $\sim L^{d}$ spins around this point that can be reversed with respect to its environment at minimum free energy cost. The droplets are the low free energy excitations upon which the theory by Fisher and Huse [3, 1 ] is based. Droplet walls have an intrinsic width of the order of the correlation length $\xi_{-}$and hence the droplet concept is naturally limited to length scales $L \gtrsim \xi_{-}(T)$. In particular, it no longer applies in the limit $T \nearrow T_{c}$, where $\xi_{-}(T)$ diverges.

Spin glasses have the property of being chaotic, as was revealed in the work of Bray and Moore [2], Fisher and Huse [3], and Banavar and Bray [13]. The term chaotic here denotes the property that the thermally averaged relative orientation of two spins a distance $L$ apart is a rapidly and randomly varying function of temperature (see figure 1). If we denote the autocorrelation interval on the temperature axis of this function by $\Delta T^{L}$, then in the ordered phase

$$
\Delta T^{L} \sim L^{-\zeta}, \quad \text { as } L \rightarrow \infty,
$$

where $\zeta$ is the chaos exponent. Upon inverting (1.1) one finds

$$
L_{\Delta T} \sim \Delta T^{-\frac{1}{\zeta}}, \quad \text { as } \Delta T \rightarrow 0,
$$

which is called the overlap length associated with a temperature difference $\Delta T$. Numerically $\zeta$ takes values between 0.75 and 1 in $1 \leqq d \leqq 3$ 14, 16, 11, 12,. Within the droplet theory the chaos phenomenon is elegantly explained as resulting from temperature changes that disturb the energy versus entropy balance of the droplet walls and thereby induce random droplet reversals. In this theory the sum $2 y+2 \zeta$ of the zero temperature thermal and chaos exponents appears to equal the fractal surface dimension $d_{s}$ of the droplet walls.

The original authors [1 3] thought that chaos was limited to length scales $L \gtrsim \xi_{-}$in the spin glass ordered phase and to dimensions where such a phase exists, i.e., above the lower critical dimension $d_{\ell}$. Ney-Nifle and Hilhorst [11,12] subsequently showed that there is chaos also in the critical region, at least for spatial dimensions $d$ in an interval extending upward from the lower critical dimension $d_{\ell} \approx 2.5$ [9,2,17] to some limit dimension $d_{+}$, estimated within MKRG to be $d_{+} \approx 3.4$ [11, 12.

The reasoning can be summarised as follows. At a length scale $L$, the critical region is the temperature interval

$$
\frac{\left|T-T_{c}\right|}{T_{c}} \lesssim L^{-y_{c}}
$$

in which $L \lesssim \xi_{-}(T)$. Here $1 / y_{c} \equiv \nu$ is the correlation length exponent. Ney-Nifle and Hilhorst use MKRG to calculate the effective couplings on scale $L$ and find that inside the critical region they vary randomly with temperature with an autocorrelation interval

$$
\Delta T^{L} \sim L^{-\zeta_{c}},
$$


where the (positive) critical point chaos exponent $\zeta_{c}$ is a new and independent exponent (see figure 1). This chaotic behaviour of the renormalised coupling $K^{L}$ (chaos with temperature) is reflected in the behaviour of the two-point correlation function $\left\langle s_{i} s_{i+L}\right\rangle$ as a function of temperature, which changes sign when the effective coupling at distance $L$ changes sign.

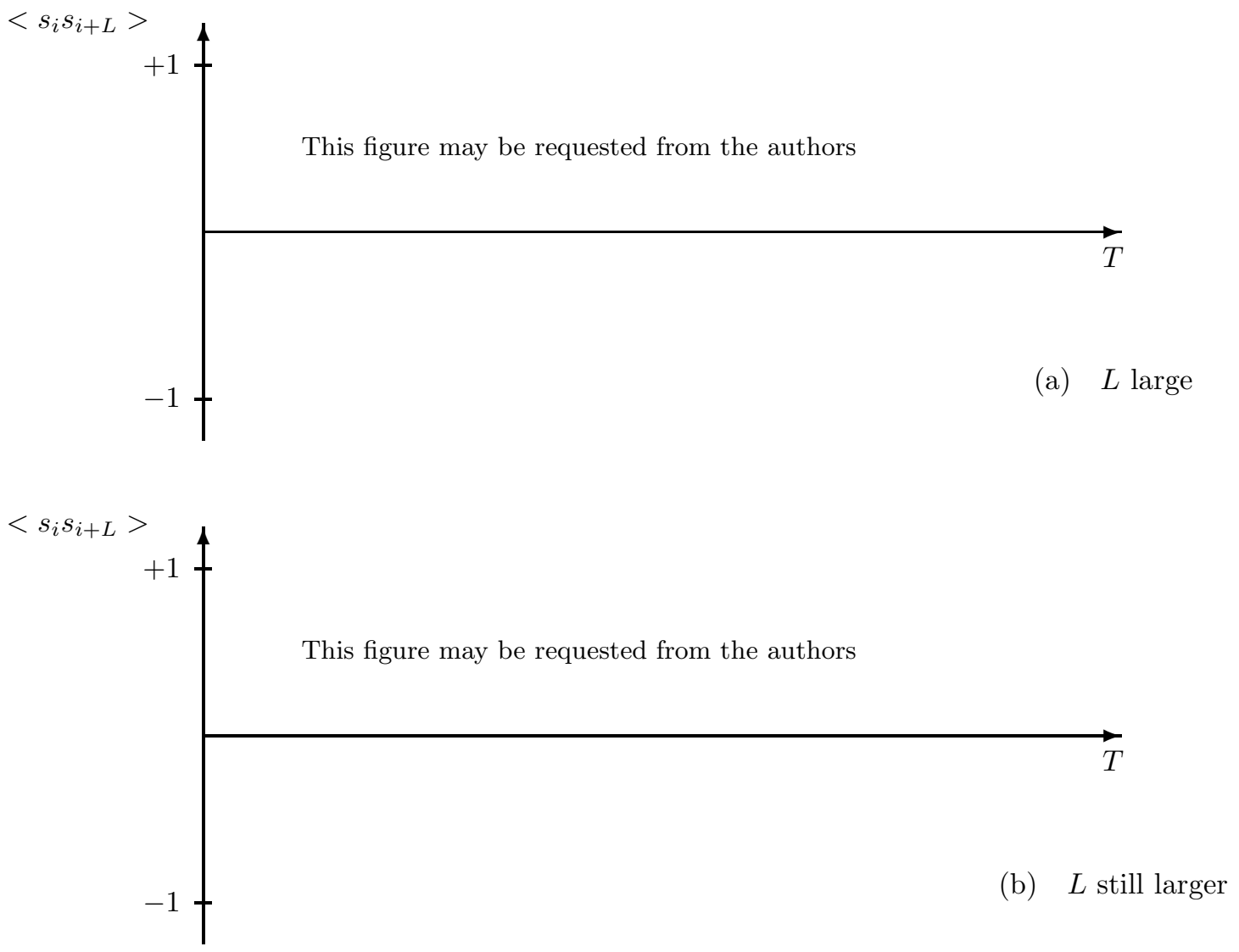

Figure 1. Temperature dependence of the two-point correlation function $\left\langle s_{i} s_{i+L}\right\rangle$ for (a) large $L$ and (b) still larger $L$. At criticality $\Delta T^{L} \sim L^{-\zeta_{c}}$ and near zero temperature $\Delta T^{L} \sim L^{-\zeta}$, for $L \rightarrow \infty$.

In view of this there is chaos inside the critical region if

$$
\zeta_{c}>y_{c}
$$

Figure 2 shows the dimension dependence of $y_{c}$ and $\zeta_{c}$. As $d \searrow d_{\ell}$, where $d_{\ell}$ is characterised by $y\left(d_{\ell}\right)=0$, these exponents join continuously their zero temperature equivalents $y(d)$ and $\zeta(d)$. The MKRG values for the critical exponents in dimension $d=3$ are [11, 12, 18.

$$
y_{c}(3) \approx 0.36, \quad \zeta_{c}(3) \approx 0.57 .
$$

Hence, according to these values, the $3 d$ spin glass is chaotic at criticality. 


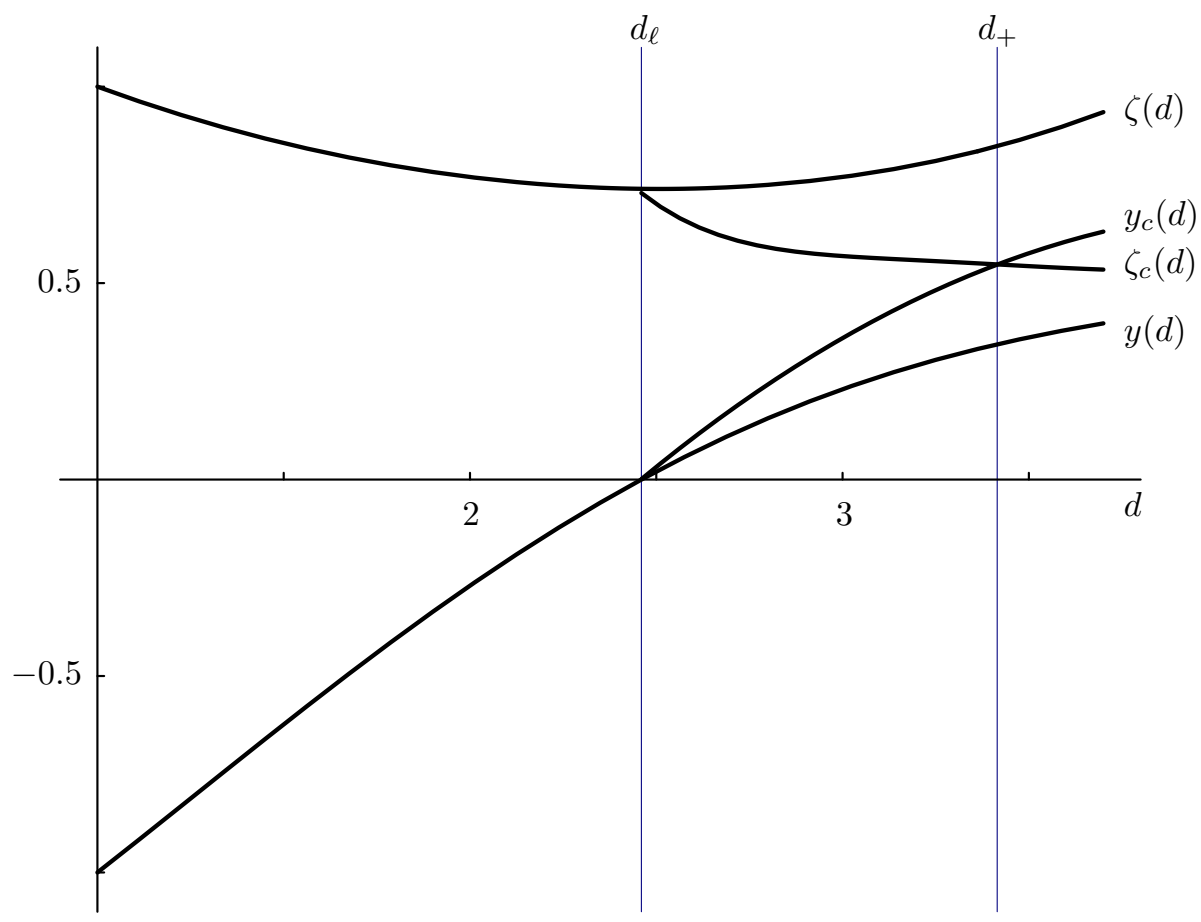

Figure 2. The critical exponents $y_{c}(d)$ and $\zeta_{c}(d)$ as a function of the dimension $d$. They join continuously the zero temperature exponents $y(d)$ and $\zeta(d)$, respectively, as $d$ approaches $d_{\ell}$ from above. This dimension is defined by $y\left(d_{\ell}\right)=0$. The intersection of the curves $y_{c}(d)$ and $\zeta_{c}(d)$ defines $d_{+}$. The curves shown are obtained from numerical evaluation of MKRG by the pool method 18 .

The problem that triggered the investigation of this paper can now be stated precisely. In view of the inapplicability of the droplet concept in the critical region, the natural question to ask is if the chaos at criticality can be given some different geometric interpretation. Blind analogy might lead one to suppose, for example, that the sum $2 y_{c}+2 \zeta_{c}$ would be equal to the fractal surface dimension of objects yet to be identified whose reversal upon temperature changes would cause the critical chaos however we shall see that this is not true. This work deals with answering the question raised above, but its results go beyond it:

The paper is organised as follows. In section II we review and extend the existing MKRG theory of the Ising spin glass. Introducing the scaling of various averaged derivatives of the renormalised coupling constant $K^{L}$, we obtain multifractal scaling of the energy-energy correlations at criticality. In section III we show how the scaling laws deduced from MKRG can be given a geometric interpretation. The fundamental entities are surfaces (of width zero) enclosing clusters of spins that may reverse with respect to their environment. The surfaces are grouped into finite interacting sets that are self-similar in the range of scales between the lattice cut-off and their own size. Such a set of interacting surfaces is appropriately called a protodroplet, since in the off-critical limit it reduces to a Fisher and Huse droplet. Near the lower critical dimension there is confluency of zero temperature and critical phenomena. In order to clarify the picture, the characteristic lengths that play a rôle are analysed in section $[\mathrm{IV}$. In section $\mathrm{V}$, based on the physical picture of section III. we establish the multifractal scaling of the spin-spin correlation function at criticality. Furthermore, the effects of a magnetic field in the ordered phase and at criticality are discussed in a unified way, within the picture of section II. We use an 
Imry-Ma type argument to deduce scaling laws for the leading behaviour of the magnetisation in the neighbourhood of the critical point, approaching $T_{c}$ from below and above. The singular situation at $d_{\ell}$ is also considered in detail. In section VI we point out the consequences of the strong spatial inhomogeneity of thermal fluctuations on the critical dynamics. The picture of this paper augmented with the additional assumption that the two-dimensional dynamics near zero temperature dynamics is activated, together with an argument of continuity, gives a first strong theoretical support for the conjecture that the critical dynamics of $d$-dimensional spin glass is activated in an interval of dimensions starting with $d_{\ell}$. In the conclusion, section VII, we mention possibilties of verifying our picture in simulations of the $d=3$ EA model.

\section{EXTENDED SCALING LAWS FOR RENORMALISED COUPLINGS}

\section{A. Introduction}

The Migdal-Kadanoff renormalisation transformation [7] was first formulated for the spin glass by Young et al [8.97. In this section we start from the same basic formulas. We briefly recall the main results known and then go on to extend some of them. Our interest concerns in particular correlations of various finds (with respect to the disorder average) between the renormalised and the unrenormalised couplings.

The MKRG works as follows. For a rescaling factor $b$ one combines a set of $b^{d}$ couplings at the $n$th level, $\left\{K_{i_{n}}^{(n)}\right\}_{i_{n+1}}$, to a new coupling, $K_{i_{n+1}}^{(n+1)}$. This is done by arranging the $K_{i_{n}}^{(n)}$ of the block $i_{n+1}$ in $b^{d-1}$ parallel strings (labeled by the index $m$ ) of $b$ couplings each. [Whenever we will indicate a RG step explicitly in thwhat follows we will relabel the couplings of $\left\{K_{i_{n}}^{(n)}\right\}_{i_{n+1}}$ as $K_{m k}^{(n)}$.] The recursion relation then reads [9,19]

$$
K_{i_{n+1}}^{(n+1)}=\sum_{m=1}^{b^{d-1}} \operatorname{arctanh} \prod_{k=1}^{b} \tanh K_{m k}^{(n)} .
$$

In the remainder we set $b=2$. The couplings $K_{i_{0}}^{(0)}$ of the originally given problem are of the form $K_{i} \equiv \frac{J_{i}}{k_{\mathrm{B}} T}$, where $i$ is a bond index, and the $J_{i}$ are the coupling constants on the original lattice with root-mean-square (rms) value $J$. We shall also use $K \equiv{\overline{K_{i}^{2}}}^{\frac{1}{2}} \equiv \beta J$ as the temperature variable. With the $J_{i}$ given, the transformation (2.1) determines uniquely the couplings at all higher levels $n=1,2, \ldots$. For the $n$ times renormalised value of $K$ we shall also write

$$
K^{L} \equiv K^{(n)} \quad \text { when } \quad L=2^{n}
$$

and for a specific scale- $L$ coupling

disc

$$
K_{R}^{L} \equiv K_{i_{n}}^{(n)}
$$

when $i_{n}$ is the label of the coupling at $\boldsymbol{R} . K^{(n)}$ can be calculated from the unrenormalised rms coupling $K \equiv K^{(0)}$ via the transformation (2.1). If, as usual, after each RG step the distribution of couplings is replaced by a Gaussian with the same variance, one finds the recursion 


$$
\begin{aligned}
K^{(n+1)} & =2^{\frac{d-1}{2}}\left[\overline{\operatorname{arctanh}^{2} \prod_{k=1}^{2} \tanh K_{m k}^{(n)}}\right]^{\frac{1}{2}} \\
& \equiv \mathcal{R}\left(K^{(n)}\right) .
\end{aligned}
$$

The transformation $\mathcal{R}$ has the following properties (for some mathematically rigorous proofs see [19] and references therein). It has the fixed points $K=0$ and $K=\infty$, and, for dimensions $d$ above $d_{\ell}$, also a critical fixed point $K=K_{c}$. Near the zero- $T$ fixed point one has

$$
K^{(n+1)} \simeq 2^{y} K^{(n)}
$$

with $y<0$ for $d<d_{\ell}$ and $y>0$ for $d>d_{\ell}$, and near the critical fixed point $K=K_{c}$

$$
K^{(n+1)}-K_{c} \simeq 2^{y_{c}}\left(K^{(n)}-K_{c}\right) .
$$

By expanding (2.1) for $\beta \rightarrow \infty$, one easily shows that closely above the lower critical dimension the equations 2.5) and (2.6) can be written together in the compact form

$$
\left(K^{L}\right)^{2}=2^{2 y}\left(K^{\frac{L}{2}}\right)^{2}-c_{0}
$$

where $c_{0}$ is a positive constant.

\section{B. Multifractal scaling at criticality}

Let $K_{R}^{L}$ be an individual renormalised coupling on scale $L$. One main result of reference [11, 12] is that in the critical region $K_{R}^{L}$ is a rapidly and randomly varying function of temperature with rms derivative

$$
\overline{\left(\frac{d K_{R}^{L}}{d K}\right)^{2}} \sim L^{2 \zeta_{c}}, \quad\left|\frac{K-K_{c}}{K_{c}}\right| \lesssim L^{-y_{c}}
$$

in an interval of dimensions $d_{\ell}<d<d_{+}$. This chaotic behaviour of the renormalised coupling $K_{R}^{L}$ is reflected in the behaviour of the two-point correlation function $\left\langle s_{i} s_{i+L}>\right.$ as a function of temperature, which changes sign when the effective coupling at distance $L$ changes sign (see figure 1). From (2.6) we have

$$
\frac{d}{d K} \overline{\left(K_{R}^{L}\right)^{2}} \sim L^{y_{c}}
$$

Upon rewriting (2.9) as

$$
\overline{K_{R}^{L} \frac{d K_{R}^{L}}{d K}} \sim L^{y_{c}}
$$

one sees that $K_{R}^{L}$ and $\frac{d K_{R}^{L}}{d K}$ are correlated, while for reasons of symmetry 


$$
\overline{\frac{d K_{R}^{L}}{d K}}=0
$$

The relations (2.8) and (2.10) imply that, at criticality $\frac{d K_{R}^{L}}{d K}$ has a large random part, proportional to $L^{\zeta_{c}}$, which has a random sign, and a small random part, proportional to $L^{y_{c}}$, whose sign is positively correlated with that of $K_{R}^{L}$ (and which is therefore, in this sense, 'systematic').

Below we shall study the joint probability distribution at criticality of $K_{R}^{L}$ and $\frac{\partial K_{R}^{L}}{\partial K_{i}} K_{i}$. We will argue that at criticality $\zeta_{c}$ is only the first of a family of an infinite number of exponents describing, together with $y_{c}$, the scaling of the moments of the joint distribution. This is in contrast to the behaviour at zero temperature whose scaling is entirely described by the exponents $y$ and $\zeta$.

Since we wish to consider two-point correlation functions in section II dependence of the renormalised coupling $K_{R}^{L}$ at a position $\boldsymbol{R}$ and at length scale $L$ on the set of unrenormalised couplings $\left\{K_{i}\right\}$ in the volume of order $L^{d}$ around $\boldsymbol{R}$. The temperature derivative of $K_{R}^{L}$ can be calculated via 2.1) as

$$
\frac{d K_{R}^{L}}{d K}=\sum_{i} \frac{\partial K_{R}^{L}}{\partial K_{i}} \frac{K_{i}}{K}
$$

where the sum is on the $L^{d}$ unrenormalised couplings. Neglecting cross terms (that contribute only to order $L^{2 y_{c}}$ as $\left.L \rightarrow \infty\right)$, we find from (2.8) and (2.12) at criticality

$$
\overline{\left(\frac{\partial K_{R}^{L}}{\partial K_{i}} K_{i}\right)^{2}} \sim L^{2 \zeta_{c}-d},
$$

Similarly 2.10$)$ and $(2.12)$ give

$$
\overline{K_{R}^{L} \frac{\partial K_{R}^{L}}{\partial K_{i}} K_{i}} \sim L^{y_{c}-d}
$$

The relations (2.13) and (2.14) are only two moments of a joint probability distribution of $K_{R}^{L}$ and $\frac{\partial K_{R}^{L}}{\partial K_{i}} K_{i}$. For the other moments at the critical point we make the ansatz

$$
\overline{\left(\frac{\partial K_{R}^{L}}{\partial K_{i}} K_{i}\right)^{2 q}} \sim L^{2 \zeta_{c}^{(q)}-d}
$$

and

$$
\overline{K_{R}^{L}\left(\frac{\partial K_{R}^{L}}{\partial K_{i}} K_{i}\right)^{2 q-1}} \sim L^{y_{c}+2 \zeta_{c}^{(q)}-2 \zeta_{c}-d}
$$

where $\zeta_{c}^{(1)} \equiv \zeta_{c}$.

Let now $K_{R}^{L} \equiv K_{i_{n}}^{(n)}$ and $K_{i} \equiv K_{i_{0}}^{(0)}$ and write by chain rule differentiation down the hierarchy of couplings

$$
\frac{\partial K_{R}^{L}}{\partial K_{i}}=\prod_{\nu=1}^{n} \frac{\partial K_{i_{\nu}}^{(\nu)}}{\partial K_{i_{\nu-1}}^{(\nu-1)}}
$$


where $i_{1}, i_{2}, \ldots$ denote the locations of the couplings on the successive levels $\nu=1,2, \ldots$ to which $K_{i}$ contributes. At criticality, the factors in the product are identically distributed random variables. If we assume them independent, then we have from (2.15) and (2.17) that at criticality

$$
2 \zeta_{c}^{(q)}-d=(\log 2)^{-1} \log \overline{\left(\frac{\partial K_{i_{1}}}{\partial K_{i_{0}}}\right)^{2 q}} .
$$

The right-hand-side of this expression can be calculated from a single renormalisation iteration after which $\zeta_{c}^{(q)}$ is known [20]. Obviously there is no reason for $\zeta_{c}^{(q)}$ to be linear in $q$. Hence we have found that at criticality there is multifractal scaling 20$]$.

The above results are valid at criticality. It is instructive to compare them to their analogues in the ordered phase. There we have for $L \gg \xi_{-}[11,12,3]$ :

$$
\begin{aligned}
& \overline{\left(\frac{d K_{R}^{L}}{d K}\right)^{2}} \sim L^{d_{s}}, \\
& \frac{d}{d K} \overline{\left(K_{R}^{L}\right)^{2}} \sim L^{2 y},
\end{aligned}
$$

where $d_{s} \equiv 2 \zeta+2 y$, hence

$$
\begin{aligned}
& \overline{\left(\frac{\partial K_{R}^{L}}{\partial K_{i}} K_{i}\right)^{2}} \sim L^{d_{s}-d}, \quad L \gg \xi_{-} . \\
& \overline{K_{R}^{L} \frac{\partial K_{R}^{L}}{\partial K_{i}} K_{i}} \sim L^{2 y-d},
\end{aligned}
$$

[We shall write $d_{s}$ throughout but note that this exponent takes the value $d_{s}=d-1$ within MKRG.] This scaling is due to the fact that bonds contribute to the energy fluctuations if and only if they are cut by a droplet wall. In the limit $T \rightarrow 0$, the derivative $\frac{\partial K_{R}^{L}}{\partial K_{i}}$ equals \pm 1 with probability $\frac{1}{2} L^{d_{s}-d}$ and vanishes otherwise [cf. (2.1) in the $\beta \rightarrow \infty$-limit, equation (3.3)]. For the same reason, we obtain for the higher moments:

$$
\begin{array}{ll}
\overline{\left(\frac{\partial K_{R}^{L}}{\partial K_{i}} K_{i}\right)^{2 q}} & \sim L^{d_{s}-d}, \\
\overline{K_{R}^{L}\left(\frac{\partial K_{R}^{L}}{\partial K_{i}} K_{i}\right)^{2 q-1}} & \sim L^{2 y-d}, \quad L \gg \xi_{-} .
\end{array}
$$

There is therefore no multifractal but only trivial scaling at length scales $L \gg \xi_{-}$in the ordered phase. With the aid of (2.15) and (2.16) we can write the moments (2.21) in the scaling form

$$
\begin{array}{ll}
\overline{\left(\frac{\partial K_{R}^{L}}{\partial K_{i}} K_{i}\right)^{2 q}} & \sim L^{2 \zeta_{c}^{(q)}-d} \mathcal{F}_{q}^{e}\left(\frac{L}{\xi_{-}}\right), \\
\overline{K_{R}^{L}\left(\frac{\partial K_{R}^{L}}{\partial K_{i}} K_{i}\right)^{2 q+1}} \sim L^{y_{c}+2 \zeta_{c}^{(q)}-2 \zeta_{c}-d} \mathcal{F}_{q}^{o}\left(\frac{L}{\xi_{-}}\right),
\end{array}
$$

with scaling functions that satisfy

$$
\begin{aligned}
& \mathcal{F}_{q}^{e}(0)=1, \\
& \mathcal{F}_{q}^{e}(x) \stackrel{x \rightarrow \infty}{\sim} x^{d_{s}-2 \zeta_{c}^{(q)}}, \\
& \mathcal{F}_{q}^{o}(0)=1, \\
& \mathcal{F}_{q}^{o}(x) \stackrel{x \rightarrow \infty}{\sim} x^{2 y-y_{c}-2 \zeta_{c}^{(q)}+2 \zeta_{c}} .
\end{aligned}
$$


In the limit $d \searrow d_{\ell}$ along the critical line $T_{c}(d)$ we know that $y_{c} \searrow 0$ and $\zeta_{c} \searrow \zeta\left(d_{\ell}\right)$. Since also ( 2.15 ) and (2.16) must then reduce to (2.21), it follows that

$$
\zeta_{c}^{(q)} \stackrel{d \unlhd}{\rightarrow} \frac{d_{\ell}\left(d_{\ell}\right)}{2}=\zeta\left(d_{\ell}\right)
$$

It appears therefore that the zero-temperature exponent $\zeta\left(d_{\ell}\right)$ fans out along the critical line into the family of the $\zeta_{c}^{(q)}$.

\section{Energy fluctuations}

We shall now exploit our extended knowledge of the MKRG to study energy-energy fluctuations. These are, generally, obtained as the appropriate derivatives of the logarithm of the partition function

$$
\log Z=\sum_{\nu=0}^{\infty} G^{(\nu)}
$$

with respect to the couplings. Here $\exp G^{(\nu-1)}$ is the multiplicative constant that appears in the partition function in the $\nu$ th renormalisation step. It is the contribution to the free energy $\beta F$ coming from excitations on a spatial scale $\ell=2^{\nu}$. Within the MKRG scheme defined by (2.1) the expression for $G^{(\nu)}$ is

$$
G^{(\nu)}=\sum_{i_{\nu+1}} g\left(\left\{K_{i_{\nu}}^{(\nu)}\right\}_{i_{\nu+1}}\right)
$$

with

$$
g\left(\left\{K_{i_{\nu}}^{(\nu)}\right\}_{i_{\nu+1}}\right)=\sum_{m=1}^{2^{d-1}} \frac{1}{2} \log \left[4 \cosh \left(K_{m 1}^{(\nu)}+K_{m 2}^{(\nu)}\right) \cosh \left(K_{m 1}^{(\nu)}-K_{m 2}^{(\nu)}\right)\right]
$$

where the sum in (2.26) runs over all blocks $i_{\nu+1}$ of $\nu$ th level couplings and we have used the convention introduced in section II to relabel the $i_{\nu}$ in an explicit RG step.

Let

$$
\Delta e_{i} \equiv e_{i}-<e_{i}>
$$

where $e_{i}=s_{i} s_{i^{\prime}}$, and $s_{i}$ and $s_{i^{\prime}}$ are neighbouring spins in the original lattice linked by a coupling $K_{i}$. The simplest energy-energy correlation function is then

$$
\beta^{2} \Gamma_{2}\left(r_{i j}\right) \equiv \beta^{2}<\Delta e_{i} \Delta e_{j}>=\sum_{\nu=0}^{\infty} K_{i} K_{j} \frac{\partial^{2} G^{(\nu)}}{\partial K_{i} \partial K_{j}} .
$$

A fourth order correlation function of interest is

$$
\beta^{4} \Gamma_{4}\left(r_{i j}\right) \equiv \beta^{2}\left[<\Delta e_{i}^{2} \Delta e_{j}^{2}>-<\Delta e_{i}^{2}><\Delta e_{j}^{2}>\right]=\sum_{\nu=0}^{\infty} K_{i}^{2} K_{j}^{2} \frac{\partial^{4} G^{(\nu)}}{\partial K_{i}^{2} \partial K_{j}^{2}} .
$$


These correlation functions are random quantities. We are now faced with the somewhat technical task of calculating averages as well as the averages of their powers. The resulting expressions are (2.34), (2.37), and (2.38), and their derivation is as follows. Let for simplicity $i=i_{0}$ and $i_{1}, i_{2}, \ldots$ denote the locations of the couplings on the successive levels $\nu=1,2, \ldots$ to which $K_{i}$ contributes and similarly for $K_{j}$. Typically, the couplings $K_{i_{\nu}}$ and $K_{j_{\nu}}$ fall in the same block when $\nu$ attains a value $\nu^{*}(i, j)$ such that

$$
2^{\nu^{*}}=r_{i j}
$$

where $r_{i j}$ is the distance between the two spins concerned. They then contribute to the same $G^{\left(\nu^{*}\right)}$ and to the same $K_{i_{\nu^{*}}}^{\left(\nu^{*}\right)}=K_{j_{\nu^{*}}}^{\left(\nu^{*}\right)}$. Working out (2.29), one finds that the second derivative leads to terms of various types. The guiding principle in finding the dominant contribution for large $r_{i j}$ is that, according to (2.15) and (2.16), averages with even powers of derivatives are larger than averages with odd powers, while some derivatives vanish due to symmetry properties of $g\left(\left\{K_{i_{\nu}}^{(\nu)}\right\}_{i_{\nu+1}}\right)$ and $K_{i_{\nu+1}}^{(\nu+1)}$. Therefore we get

$$
\begin{aligned}
\beta^{2} \Gamma_{2}\left(r_{i j}\right) \simeq & \frac{\partial^{2} G^{\left(\nu^{*}\right)}}{\partial K_{i_{\nu^{*}}} \partial K_{j_{\nu^{*}}}} K_{i} \frac{\partial K_{i_{\nu^{*}}}}{\partial K_{i}} K_{j} \frac{\partial K_{j_{\nu^{*}}}}{\partial K_{j}}+ \\
& +\sum_{\nu=n^{*}+1}^{\infty} \frac{\partial^{2} G^{(\nu)}}{\partial K_{i_{\nu}}^{2}} \prod_{\mu=\nu^{*}+1}^{\nu-1}\left(\frac{\partial K_{i_{\mu+1}}}{\partial K_{i_{\mu}}}\right)^{2} \frac{\partial^{2} K_{i_{\nu^{*}+1}}}{\partial K_{i_{\nu^{*}}} \partial K_{j_{\nu^{*}}}} K_{i} \frac{\partial K_{i_{\nu^{*}}}}{\partial K_{i}} K_{j} \frac{\partial K_{j_{\nu^{*}}}}{\partial K_{j}}
\end{aligned}
$$

Let $\beta^{2} \Gamma_{2}^{L}\left(r_{i j}\right)$ be the term with $\nu=n$ in $(2.29)$, i.e., it is the contribution of scale $L$ fluctuations to $\beta^{2} \Gamma_{2}\left(r_{i j}\right)$. Obviously, $\Gamma_{2}^{L}(r)$ vanishes for $L<r$. Using again 2.16$)$ we arrive at

$$
\overline{\Gamma_{2}^{L}(r)} \sim\left(\frac{r}{L}\right)^{d-2 \zeta_{c}} \frac{1}{r^{2 d-2 y_{c}}}, \quad L \geqq r,
$$

for the contribution of the energy fluctuations due to two spins a distance $r$ apart in a volume of linear size $L \geqq r$. Hence, summing over length scales, we get the energy-energy correlation function at distance $r$ :

$$
\overline{\Gamma_{2}(r)}=\tilde{\sum_{L \geqq r}} \overline{\Gamma_{2}^{L}(r)} \sim \frac{1}{r^{2 d-2 y_{c}}},
$$

the sum being dominated by the first term.

If one works out the expression (2.30) for the fourth cumulant, the fourth derivative leads again to terms of various types. In order to find the dominant contribution for large $r_{i j}$ we proceed as above. The result is

$$
\begin{aligned}
\beta^{4} \Gamma_{4}\left(r_{i j}\right) \simeq & \frac{\partial^{4} G^{\left(\nu^{*}\right)}}{\partial^{2} K_{\nu_{\nu^{*}}} \partial^{2} K_{j_{\nu^{*}}}} K_{i}^{2}\left(\frac{\partial K_{i_{\nu^{*}}}}{\partial K_{i}}\right)^{2} K_{j}^{2}\left(\frac{\partial K_{j_{\nu^{*}}}}{\partial K_{j}}\right)^{2} \\
& +\sum_{n=\nu^{*}+1}^{\infty} \frac{\partial^{4} G^{(\nu)}}{\partial K_{i_{\nu}}^{4}} \prod_{\mu=\nu^{*}+1}^{\nu-1}\left(\frac{\partial K_{\mu_{\mu+1}}}{\partial K_{i_{\mu}}}\right)^{4} \frac{\partial^{4} K_{i_{\nu^{*}+1}}}{\partial K_{i_{\nu^{*}}}^{2} \partial K_{j_{\nu^{*}}}^{2}} K_{i}^{2}\left(\frac{\partial K_{i_{\nu^{*}}}}{\partial K_{i}}\right)^{2} K_{j}^{2}\left(\frac{\partial K_{j_{\nu^{*}}}}{\partial K_{j}}\right)^{2} .
\end{aligned}
$$

The quantity $\beta^{4} \Gamma_{4}^{L}\left(r_{i j}\right)$, defined as the term with $\nu=n$ in equation (2.30), vanishes for $L<r_{i j}$. For $L \geqq r$, averaging on the disorder and using (2.15) gives

$$
\overline{\Gamma_{4}^{L}(r)} \sim\left(\frac{r}{L}\right)^{d-2 \zeta_{c}^{(2)}} \frac{1}{r^{2 d-4 \zeta_{c}}} .
$$


Integrating this equation over length scales $L$ as above, one finds

$$
\overline{\Gamma_{4}(r)}=\tilde{\sum_{L \geqq r}} \overline{\Gamma_{4}^{L}(r)} \sim \frac{1}{r^{2 d-4 \zeta_{c}}},
$$

because $d-2 \zeta_{c}^{(2)}$ is the (necessarily positive) exponent of a moment of a probability distribution disc [cf. also 2.15) and (2.24)].

By taking powers of (2.32) and averaging with the help of (2.15) and (2.16), one finds

$$
\begin{aligned}
& \overline{\Gamma_{2}^{2 q}(r)} \sim r^{4 \zeta_{c}^{(q)}-2 d}, \\
& \overline{\Gamma_{2}^{2 q-1}(r)} \sim r^{2 y_{c}+4 \zeta_{c}^{(q)}-4 \zeta_{c}-2 d},
\end{aligned}
$$

for the moments of the correlation function $\Gamma_{2}(r)$. Analogous expressions hold for the moments of the higher order correlation functions. Since $\zeta_{c}^{(q)}$, given by (2.18), is a nonlinear function of $q$, this implies multifractal scaling of the energy-energy correlations at criticality.

By summing finally (2.34) on all $i$ and $j$ in a volume $V$, one obtains the mean square fluctuation of the total energy in that volume,

$$
\frac{\overline{<\Delta E^{2}>}}{V} \sim \int_{\sim 1}^{V} d r r^{d-1} \overline{\Gamma_{2}(r)} \sim k_{\mathrm{B}} T_{c}^{2} C_{V}\left(T_{c}\right), \quad V \rightarrow \infty,
$$

valid if $d-2 \zeta_{c}>0$, which is the case in an interval upward from $d_{\ell}$ [cf. (2.15) and (2.24)]. By summing (2.37) one gets similarly the fourth cumulant of the energy in a volume $V$,

$$
\frac{\overline{\left\langle\Delta E^{4}\right\rangle_{\text {cum }}}}{V} \equiv \frac{\overline{\left\langle\Delta E^{4}>\right.}-3 \overline{\left.<\Delta E^{2}\right\rangle^{2}}}{V}
$$

The result depends on the sign of $4 \zeta_{c}-d$ and reads

$$
\begin{aligned}
& \frac{\overline{\left\langle\Delta E^{4}\right\rangle_{\mathrm{cum}}}}{V} \sim \int_{1}^{V^{\frac{1}{d}}} d r r^{d-1} \overline{\Gamma_{4}(r)} \\
& V \rightarrow \infty\left\{\begin{array}{rlr}
V^{4 \frac{\zeta_{c}}{d}-1} & \text { if } \quad 4 \zeta_{c} / d>1 . \\
\text { cst. } & \text { if } \quad 4 \zeta_{c} / d<1 .
\end{array}\right.
\end{aligned}
$$

Equation (2.41a) is likely to hold in a narrow range of dimensions upward from $d_{\ell}$, and equation $(2.41 \mathrm{~b})$ for higher dimensions. Equations 2.39 ) and (2.41 $\mathrm{b}$ ) have nonanalytic correction terms in $V$. In nonrandom thermodynamic systems the energy cumulants tend to constants in the thermodynamic limit. Equation (2.41a), in contrast, expresses an anomalous scaling of the fourth cumulant with volume. It is easy to check that the same is true for the higher order cumulants. Such an anomalous scaling of the energy cumulants in spin glass was first pointed out by $\mathrm{FH}$ for the ordered phase [see [3], equations (7.11) and (7.12)]. The basic reason for this anomalous behaviour of the cumulants is that the energy fluctuations are collective, even beyond the scale $\xi_{-}$, up to scale infinity, and involve larger and larger energy differences. 


\section{GEOMETRIC INTERPRETATION OF THE CRITICAL SCALING LAWS}

Our purpose is now to understand the scaling behaviour found in the previous section in terms of a geometric picture. In the ordered phase, for length scales $L \gg \xi_{-}$, the work of Fisher and Huse 3. 1 has made it clear how to pass from MKRG to such a picture. After recalling how this is done, in section Md, we will address the same issue in the critical region, in section $\amalg$ II .

\section{A. MKRG and the droplet picture of the ordered phase}

\section{Droplets}

At length scales $L \gg \xi_{-}$the block spins of the MKRG procedure are naturally identified with the droplets of the FH picture. Thermally active block spins, i.e., block spins coupled to their environment with energies less than $k_{\mathrm{B}} T$, then represent thermally active droplets. The reversal energy of a scale- $L$ block spin is equal to twice the sum of the couplings $K_{R}^{L}$ that link it to its environment (with signs equal to the values of the neighbouring block spins). We shall speak of the $K_{R}^{L}$ as the excitation free energies of collective spin reversals on scale $L$, even though strictly speaking it is always the sum of a few $K_{R}^{L}$ that is involved.

The probability distribution of the $K_{R}^{L}$ scales with $L$ in the same way as the probability distribution $P_{L}$ of the reversal free energies $\Delta F_{R}^{L}$ of the $\mathrm{FH}$ droplets, viz. as

$$
P^{L}\left(\Delta F_{R}^{L}\right) \simeq \frac{1}{\Upsilon(T) L^{y}} \mathcal{P}\left(\frac{\Delta F_{R}^{L}}{\Upsilon(T) L^{y}}\right), \quad L \rightarrow \infty .
$$

Here $\Upsilon(T)$ is the temperature dependent stiffness coefficient of the ordered phase with $\Upsilon(0) \sim J$ and $\Upsilon(T)^{T} \beth^{\tau^{T}} \xi_{-}^{-y}$ 16,3]. Equation (3.1) implies in particular that the probability $f^{L}(T)$ for a scale $L$ block spin to be thermally active is

$$
f^{L}(T) \sim \frac{k_{\mathrm{B}} T}{\Upsilon(T) L^{y}}, \quad L \gg \xi_{-} .
$$

Therefore in the large- $L$ limit the thermally active scale $L$ block spins become infinitely dilute and may be considered as independent degrees of freedom.

FH argue that the droplet wall has a fractal dimension $d_{s}$ with $d-1<d_{s}<d$. At low enough temperature the droplet wall will be of zero width and cut through a well-defined set of couplings of the unrenormalised system. The associated reversal energy is the sum of the energies of each of the couplings cut. The number of couplings cut will grow typically as $L^{d_{s}}$. MKRG gives $d_{s}=d-1$. This is easily seen if one expands the elementary renormalisation step (2.1) in the $\beta \rightarrow \infty$ limit. It reads

$$
K_{i_{n+1}}^{(n+1)}=\sum_{m=1}^{2^{d-1}} \operatorname{sgn}\left(K_{m 1}^{(n)} K_{m 2}^{(n)}\right) \min \left(\left|K_{m 1}^{(n)}\right|,\left|K_{m 2}^{(n)}\right|\right)
$$

at $T=0$. Upon differentiating (3.3) with respect to the $2^{d}$ couplings that enter into the expression one finds that, at zero temperature, the derivatives with respect to $2^{d-1}$ of them are equal to \pm 1 , 
whereas the others vanish. After $n$ renormalisation steps with $2^{n}=L$ one sees that the scale- $L$ block spin corresponds to a droplet with wall area $\sim L^{d-1}$. This special value for $d_{s}$, i.e., $d_{s}=d-1$, must nevertheless be ascribed to the particular choice of the MKRG recursion relations and is not fundamental. Numerical work 2124 points to nontrivial values of $d_{s}$ and one can envisage an improved MKRG that would lead to $d_{s} \neq d-1$. The wall of a droplet at finite temperature has an intrinsic width of the order of the correlation length $\xi_{-}$(see figure 3). But since the RG flow carries the system to the zero temperature fixed point, the droplet walls coarse grained to at least the scale $\xi_{-}$have the same fractal dimension $d_{s}$ at length scales $L \gg \xi_{-}$.

This figure may be requested from the authors

Figure 3. A scale- $L$ droplet at finite temperature $\left(L \gg \xi_{-}\right)$. The wall of a droplet at finite temperature has an intrinsic width of the order of the correlation length $\xi_{-}$. In the large- $L$ limit, scale- $L$ thermally active droplets get more and more dilute and therefore may be treated as noninteracting. By the same token, it consists of one and only one main connected domain of order $L^{d}$ spins, whose wall width is negligible with respect to its own linear size. Therefore droplets are two-level systems, with "up" and "down" referring to the main domain.

In the large- $L$ limit, scale- $L$ thermally active droplets get more and more dilute and therefore may be treated as noninteracting. By the same token, upon assuming that there are no correlations between the spatial positions of thermally active droplets of different scales, one may neglect interactions between scale- $L$ droplets and droplets on scales just below (e.g., $L / 2$ and $L / 4$ ). As one goes down further in scale, however, droplet-droplet interactions will at some point come into play, as may be estimated as follows. On some arbitrary linear scale $\ell<L$, the wall of a scale- $L$ droplet will pass through $(L / \ell)^{d_{s}}$ blocks of size $\ell$ of which typically a number $f^{\ell}(T)(L / \ell)^{d_{s}}$ will contain thermally active droplets interacting with the wall. For $L$ below a value that following FH [3] we shall call $\lambda_{L}$, this number will typically be larger than unity and the wall of the scale- $L$ droplet is "interaction widened" up to the scale $\lambda_{L}$. An expression for $\lambda_{L}$ can readily be found [3] but of interest here is only the property

$$
\lim _{L \rightarrow \infty} \frac{\lambda_{L}}{L}=0
$$

It implies that for large $L$ a droplet consists of one and only one main connected domain of order 
$L^{d}$ spins, whose wall width is negligible with respect to its own linear size. Therefore droplets are two-level systems, with "up" and "down" referring to the main domain.

\section{Interaction between droplets}

How does the interaction between droplets of different scales appear in the MKRG formalism ? To answer that question we consider a string of two scale- $L$ couplings $K_{1}^{(n)}$ and $K_{2}^{(n)}$ (contributing to a higher level coupling $K_{i_{n+1}}^{(n+1)}$ ) with in the center a scale- $L$ block spin $S_{0}$ and at the ends two larger scale block spins $S_{1}$ and $S_{2}$ (one of them is necessarily a scale- $2 L$ spin and the other one belongs to a scale $\geqq 4 L$ ). The derivative of (3.3) with respect to a scale- $L$ coupling $K_{m k}^{(n)}$ is bounded by

$$
0<\left|\frac{\partial K_{i_{n+1}}^{(n+1)}}{\partial K_{m k}^{(n)}}\right|<1 .
$$

The preceding discussion implies that when its absolute value is not close to the lower or upper limit values 0 or 1 , respectively, this is the signature of thermal activity. If this is true for the derivative with respect to $K_{1}^{(n)}$ or $K_{2}^{(n)}$, then the wall between the droplets $S_{1}$ and $S_{2}$ may cut either bond of the string. The two positions of the wall differ by the orientation of $S_{0}$. Hence the droplet $S_{0}$ thermally decorates the wall between the droplets $S_{1}$ and $S_{2}$. The two wall positions will in general have different weights. Upon working out the derivatives of interest with the aid of (2.1) we find

$$
\frac{\partial K_{i_{n+1}}^{(n+1)}}{\partial K_{i}^{(n)}}=\frac{\frac{1}{2} \sinh \left(2 K_{i}^{(n)}\right)}{\cosh \left(K_{1}^{(n)}+K_{2}^{(n)}\right) \cosh \left(K_{1}^{(n)}-K_{2}^{(n)}\right)}, \quad i=1,2 .
$$

The two derivatives are of comparable absolute value, and hence thermal activity is nonnegligible, only when

$$
\left|K_{1}^{(n)} \pm K_{2}^{(n)}\right| \lesssim \frac{k_{\mathrm{B}} T}{J}
$$

The \pm sign corresponds to the two possible relative orientations of $S_{1}$ and $S_{2}$. When (3.7) is satisfied for one of the two signs, it will not be, in general, for the other sign. This is the "interaction" between droplets of different scales. This feature of the MKRG also appears in the FH droplet picture: a scale $\ell$ droplet lies on the wall of a scale $L$ droplet $(L>\ell)$, has a wall section of linear size $\ell$ in common with it, and is thermally active for one, but not for the other orientation of the large droplet.

At low critical temperatures the derivative (3.6) has a probability distribution on the interval $[-1,1]$ which strongly peaks at 0 and \pm 1 and is small elsewhere. At $T=0$, it is the sum of three delta peaks at 0 and \pm 1 . Since the ordered phase is controlled by the zero temperature fixed point, the phenomenon of interaction widening discussed here plays no rôle for the large scale properties in the spin glass ordered phase. It is important, however, for the understanding of the crossover from zero temperature to criticality, as we shall see below. 


\section{B. The low-dimensional critical region - protodroplets}

\section{Surfaces}

In the critical region, that is, on length scales $L$ below the correlation length and whether below or above $T_{c}$, the FH droplets cannot be defined. Yet, in the MKRG picture thermally active block spins can be defined as before, viz. by the condition that their interaction energy with the environment be less than some constant times $k_{\mathrm{B}} T$.

Let us consider for definiteness the critical line in the $T$ - $d$ plane. On this line the probability for a block spin to be thermally active is of the order of $k_{\mathrm{B}} T_{c} / J \equiv p>0$, whatever the spatial scale $L$. Therefore there must be interactions between these spins whose importance is characterised by the parameter $p$. Similarly, from the fact that along the critical line the derivatives (3.6) have a nontrivial probability distribution it follows that there are interactions between thermally active block spins of neighbouring scales $(L$ and $2 L)$.

Block spins can no longer be identified with droplets and we should look for a new interpretation. The key to this interpretation is to consider the limit $p \rightarrow 0$. For $d$ very close to $d_{\ell}$ the scale independent probability $p$ for a block spin to be thermally active is very small. Also in this limit the probability law for the derivatives (3.6) approaches the trivial three-peak distribution, which means that the interaction between block spins of neighbouring scales disappears.

Let us now look at the unrenormalised lattice (scale $L=1$ ) for $p$ extremely small. The thermally active spins will be extremely dilute so that for the moment we may ignore the interactions in the discussion. We could indicate the thermally active spins in a figure by surrounding them with a surface. For $L$ not too large we now identify a thermally active scale- $L$ block spin with a size- $L$ surface that encloses a cluster of unrenormalised spins that may reverse collectively with respect to their environment due to thermal agitation. These larger surfaces may be irregularly shaped. The essential point is that the surfaces of the individual thermally active spins as well as of the thermally active clusters are of width zero, because not interacting with any other thermally active degree of freedom. These thermally active surfaces are systems with two energy levels. They may be considered as independent degrees of freedom under certain circumstances, e.g., in the limit $p \ll 1$ and/or for not too large values of $L$, or for the calculation of the spin-spin correlation function (see section $\mathrm{VA}$ ).

\section{Interaction between surfaces}

We now ask about the effect of interactions between the surfaces. Interactions occur due to surface sections that they have in common. In the small- $p$ limit, the thermally active surfaces occupy random positions in space. Let us consider two scales $L$ and $\ell<L$. If $\bar{d}\left(T_{c}\right)$ is the surface fractal dimension, then a size- $L$ surface will pass through $(L / \ell)^{\bar{d}\left(T_{c}\right)}$ regions of linear size $\ell$ and interact typically with a number $p(L / \ell)^{\bar{d}\left(T_{c}\right)}$ of size $\ell$ thermally active surfaces. This number becomes $\gtrsim 1$ for

$$
L \gtrsim L_{\mathrm{entr}} \equiv\left(\frac{1}{p}\right)^{\frac{1}{\bar{d}\left(T_{c}\right)}} \sim\left(\frac{J}{k_{\mathrm{B}} T_{c}}\right)^{\frac{1}{\bar{d}\left(T_{c}\right)}}
$$


Hence beyond this length scale the surface entropy plays a rôle, i.e., the thermally active surfaces are likely to interact with smaller size thermally active surfaces with which they then form what we shall call composite thermally active surfaces.

The probability for a thermally active surface to interact with another one of size comparable to its own is small (of order $p$ ), but is also taken into account at this level of approximation. We should therefore ask if the interactions do not lead to a coupling, direct or indirect, between all thermally active degrees of freedom in the lattice. It is not difficult to estimate that for small enough $p$ the interactions couple the thermally active degrees of freedom only into finite disjoint sets. We call such a set an isolated thermally active composite surface (see figure 4). An appropriate name which is much shorter is a protodroplet, as will appear in subsection III C.

This figure may be requested from the authors

Figure 4. A scale- $L$ protodroplet in the critical region. The composite thermally active surfaces are many-level systems of coupled degrees of freedom. Since the composing surfaces may be of comparable size, the protodroplet cannot be simply characterised by a single up/down variable. It is a hierarchical object, composed of interacting two-level systems - the surfaces - which is statistically self-similar up to the scale of its own size. The energy fluctuations are due to thermally active bonds between adjacent spins that are cut by the surfaces, as indicated in the figure. The multifractality of the energy correlations is a consequence of the nontrivial distribution of relative weights between the two possible orientations of each composing surface.

The composite thermally active surfaces are many-level systems of coupled degrees of freedom. Since the composing surfaces may be of comparable size, the protodroplet cannot be simply characterised by a single up/down variable. It is a hierarchical object, composed of interacting two-level systems - the surfaces - which is statistically self-similar up to the scale of its own size. Its multifractality is a consequence of the nontrivial distribution of relative weights between the two possible orientations of each composing surface. If the above argumentation, starting from thermally active surfaces and leading to the identification of protodroplets, is supplemented with appropriate scaling hypotheses, it may be seen as an alternative - valid in the limit $d \searrow d_{\ell}$ - to the MKRG formalism in providing a basis for the theory of the low-dimensional critical state. 


\section{Summary}

On the critical line $T_{c}(d)$ in the temperature-dimension plane the spin glass system is statistically invariant under scale transformations, and has thermal fluctuations on all length scales. The equilibrium ensemble is not dominated by a single representative configuration or class of configurations, which at first sight renders a geometric analysis forbiddingly difficult. Such an analysis is nevertheless possible, for sufficiently low $T_{c}(d)$, i.e., for $d$ close to $d_{\ell}$. The simplifying feature is that at low temperatures, and whether the system is critical or not, the fraction of couplings that effectively participate in the energy fluctuations goes to zero. The thermally active couplings therefore determine a set of surfaces of low spatial density. Each surface encloses a cluster of spins which may reverse with respect to a frozen local environment in the ordered phase as well as at criticality.

What has been said in section $\amalg I \mathrm{~B}$ holds at $T=T_{c}$ as well as in the whole critical region. Imagine now that for some $T$ below $T_{c}$ we increase the length scale $L$. The probability for the protodroplet to be composed of two comparable size surfaces will go down from its value $p$ for $L \lesssim \xi_{\text {- }}$ to zero for $L \rightarrow \infty$. For $L \gtrsim \xi_{-}$a single surface will dominate and the protodroplet has become a droplet. Hence the concept of protodroplets generalises the concept of droplets in dimensions near $d_{\ell}$ and permits a geometrical picture that also applies to the critical region. Random reversals of protodroplets are at the origin of chaos in the ordered phase as well as at criticality.

\section{CHARACTERISTIC LENGTHS NEAR THE LOWER CRITICAL DIMENSION}

Near the lower critical dimension there is a confluency of zero-temperature and critical phenomena. In order to clarify the picture, we analyse in this subsection the characteristic lengths that play a rôle, in particular the correlation length $\xi_{-}$and the overlap length $L_{\Delta T}$ defined in ( 1.2).

\section{A. Correlation length near $d_{\ell}$}

The correlation length above and below $T_{c}$ is determined by the $\mathrm{RG}$ transformation $\mathcal{R}(K)$, whose explicit form near $d_{\ell}$, equation (2.7), can also be written as

$$
K^{2 L}=2^{y} K^{L}-c_{0}\left(K^{L}\right)^{-1}+\cdots,
$$

where $c_{0}$ is a positive constant. For further analysis it is convenient to pass to continuous $L$, which gives

$$
\frac{d K^{L}}{d \log L}=y K^{L}-c\left(K^{L}\right)^{-1}+\cdots
$$

with $c$ a positive constant. At low temperatures we may neglect the dot terms in (4.2). Above $d_{\ell}$, the exponent $y(d)$ is positive and equation (4.2) has the two fixed-point solutions $K^{L}=\infty$ and $K^{L}=\sqrt{c / y} \equiv K_{c}$. The latter relation can be recast as

$$
y=c\left(\frac{k_{\mathrm{B}} T_{c}}{J}\right)^{2} .
$$


Since $y$ is linear in $d-d_{\ell}$, this shows that $T_{c} \sim \sqrt{d-d_{\ell}}$ just above $d_{\ell}$ [9,3]. At low temperatures, the solution of (4.2) for initial value $K^{(0)}=K=\frac{J}{k_{\mathrm{B}} T}$ is

$$
\frac{\left(K^{L}\right)^{2}}{K_{c}^{2}}-1=\left(\frac{K^{2}}{K_{c}^{2}}-1\right) L^{2 y}
$$

This shows that just above $d_{\ell}$ the critical point exponent $y_{c}$ is equal to $2 y$, as also found by [1, 3]. Correlation lengths $\xi_{+}(T)$ above $T_{c}$ and $\xi_{-}(T)$ below $T_{c}$ may be defined by

$$
\begin{aligned}
& \left(K^{\xi_{+}}\right)^{-1}=f_{+}, \\
& \left(K^{\xi_{-}}\right)^{-1}=f_{-} K_{c}^{-1},
\end{aligned}
$$

where $f_{+}$is a fixed constant often set equal to unity and $f_{-}$a fixed constant less than 1 . These are the usual definitions; in particular, at $\left(K^{\xi_{-}}\right)^{-1}=f_{-} K_{c}^{-1}$ the renormalisation group crosses over from critical to zero temperature fixed point control. From (4.4) and (4.5) we find

$$
\begin{array}{ll}
\xi_{+}=\left[1-\left(\frac{k_{\mathrm{B}} T_{c}}{f_{+} J}\right)^{2}\right]^{\frac{1}{2 y}}\left[1-\left(\frac{T_{c}}{T}\right)^{2}\right]^{-\frac{1}{2 y}}, \quad & T>T_{c}, \\
\xi_{-}=\left[\frac{1}{f_{-}^{2}}-1\right]^{\frac{1}{2 y}}\left[\left(\frac{T_{c}}{T}\right)^{2}-1\right]^{-\frac{1}{2 y}}, & T<T_{c} .
\end{array}
$$

Some comments are in place. Using (4.3), we find that for $d \searrow d_{\ell}$ the coefficient of $\xi_{+}$has the well-defined limit

$$
\left[1-\left(\frac{k_{\mathrm{B}} T_{c}}{f_{+} J}\right)^{2}\right]^{\frac{1}{2 y}} \sim e^{\frac{J^{2}}{2 c\left(k_{\mathrm{B}} T_{c}\right)^{2}} \log \left(1-\frac{\left(k_{\mathrm{B}} T_{c}\right)^{2}}{\left(f_{+} J\right)^{2}}\right)} \simeq e^{-\frac{1}{2 c f_{+}{ }^{2}}}, \quad d \searrow d_{\ell} .
$$

Hence the definition (4.5) fixes $\xi_{+}$near $d_{\ell}$ up to a finite multiplicative constant. At $d_{\ell}$, we have

$$
\xi_{+}(T) \simeq e^{-\frac{1}{2 c f_{+}{ }^{2}}+\frac{J^{2}}{2 c\left(k_{\mathrm{B}} T\right)^{2}}}
$$

as also found by McMillan [1] and Bray and Moore [25]. If, however, we try to take the limit $d \searrow d_{\ell}$ with $\frac{T}{T_{c}}$ fixed in the expression for $\xi_{-}$, we find

$$
\lim _{\substack{d \searrow d_{\ell} \\
\frac{T}{T_{c}} \text { fixed }}} \xi_{-}(T)=\lim _{y \searrow 0}\left(\frac{\frac{1}{f_{-}^{2}}-1}{\frac{T_{c}^{2}}{T^{2}}-1}\right)^{\frac{1}{2 y}}=\left\{\begin{array}{ll}
\infty, & f_{-}<\frac{T}{T_{c}} \\
0, & \frac{T}{T_{c}}<f_{-}
\end{array},\right.
$$

so that the limit value of $\xi_{-}$at $\frac{T}{T_{c}}$ fixed is 0 or $\infty$ depending on the arbitrary constant $f_{-}$. Of course, in the $T$ - $d$ plane, the point $\left(T=0, d=d_{\ell}\right)$ lies on the intersection of two lines, with $\xi_{-}=0$ and $\xi_{-}=\infty$ so that $\xi_{-}(T, d)$ has a mathematical singularity in $\left(0, d_{\ell}\right)$. Nevertheless this feature is insignificant if one keeps $d$ fixed. At fixed $d$, equation (4.5) remains sensible. Equation (4.9) only indicates that the range of length scales $L$ at which one is uncertain whether being below or above $\xi_{-}$ becomes larger upon lowering the dimension towards $d_{\ell}$. 


\section{B. Chaos near the lower critical dimension}

Chaos in the critical region has first been shown to exist by a RG analysis in which the density of zeros of a scale- $L$ coupling on the temperature axis was considered. In this subsection we will illucidate in more detail the critical chaos near the lower critical dimension and show how it is reflected in the change in activity of the thermal degrees of freedom.

\section{The density of zeros of a scale- $L$ coupling on the $T$-axis}

It is of interest to consider the autocorrelation function $C^{L}(T, T+\Delta T)$ on the temperature axis of a renormalised coupling at scale $L$. For this quantity, normalised to 1 at $\Delta T=0$, one expects the behaviour

$$
1-C^{L}(T, T+\Delta T) \simeq \frac{1}{2} \Delta T^{2} \rho_{L}^{2}(T), \quad \text { as } \Delta T \rightarrow 0
$$

where $\rho_{L}(T)$ is the average density of zeros of such a coupling. This quantity is, in principle, determined when a renormalisation group transformation is given.

Below we will calculate $\rho_{L}(T)$ near the lower critical dimension. Within the approximation of the Migdal-Kadanoff renormalisation group, Ney-Nifle and Hilhorst 11, 12] found an expression for $\rho_{L}(T)$ which slightly rewritten reads

$$
T^{2} \rho_{L}^{2}(T)=K^{2} \sum_{j=0}^{n-1}\left(\frac{d K^{(j)}}{d K}\right)^{2} \mu\left(K^{(j)}\right) \lambda\left(K^{(j+1)}\right) \ldots \lambda\left(K^{(n-1)}\right) .
$$

Here, as before, $K=\frac{J}{k_{\mathrm{B}} T}$; the functions $\lambda$ and $\mu$ are determined by the renormalisation group with $\lambda(K) \equiv 2^{z(K)}$ increasing from $2^{\zeta_{c}}$ to $2^{\zeta}$ in the interval $\left(K_{c}, \infty\right)$ and $\mu(K)$ behaving as $\mu(K) \simeq \frac{\mu_{\infty}}{K^{5}}$ for $K \rightarrow \infty$, where $\mu_{\infty}$ is a constant whose MKRG value is $\approx 0.12$.

Passing to a continuous length scale and using (4.4) for the $K^{(i)}$ gives

$$
T^{2} \rho_{L}^{2}(T)=\frac{\mu_{\infty}}{\log 2} \frac{K^{4}}{K_{c}^{7}} \int_{1}^{L} \frac{d \ell}{\ell}\left[1+\left(\frac{K^{2}}{K_{c}^{2}}-1\right) \ell^{2 y}\right]^{\frac{7}{2}} \exp \left(2 \int_{\ell}^{L} \frac{d \tilde{\ell}}{\tilde{\ell}} z\left(K^{\tilde{\ell}}\right)\right)
$$

In the limit of interest we can expand $z(K)$ around $K=\infty$ in powers of $K^{-1}$. From equation (5.5a) of 12] we can deduce that $z$ is even in $K$ so that to lowest nontrivial order

$$
z(K)=\zeta-\frac{1}{2} \zeta^{\prime \prime} K^{-2}
$$

Using (4.13) and again (4.4), and setting $\tau^{-1} \equiv \frac{T_{c}^{2}}{T^{2}}-1$, we obtain

$$
\rho_{L}^{2}(T) \stackrel{d}{\beth^{\searrow}} d_{\ell} \frac{\mu_{\infty}}{\log 2}\left(\frac{k_{\mathrm{B}}}{J}\right)^{3} \frac{T_{c}^{7}}{T^{6}} \int_{1}^{L} \frac{d \ell}{\ell}\left[1+\tau^{-1} \ell^{2 y}\right]^{\frac{7}{2}} \exp \left(2 \int_{\ell}^{L} \frac{d \tilde{\ell}}{\tilde{\ell}}\left[\zeta-\frac{1}{2} \zeta^{\prime \prime} K_{c}^{-2}\left[1+\tau^{-1} \tilde{\ell}^{2 y}\right]^{-1}\right]\right) .
$$


Changing variables to $s \equiv \frac{\ell^{2 y}}{\tau}$, we get finally for the average density of zeros on the temperature axis

$$
\rho_{L}^{2}(T) \stackrel{d \beth^{d}}{d_{\ell}} \frac{\mu_{\infty}}{\log 2}\left(\frac{k_{\mathrm{B}}}{J}\right)^{3} \frac{T_{c}^{7}}{T^{6}}\left(\frac{L^{2 y}}{\tau}\right)^{\frac{\zeta}{y}}\left(1+\frac{\tau}{L^{2 y}}\right)^{\frac{\zeta^{\prime \prime}}{2 c}} I_{y}\left(\frac{L^{2 y}}{\tau}, \frac{1}{\tau}\right)
$$

with

$$
I_{y} \equiv \frac{1}{2 y} \int_{\frac{1}{\tau}}^{\frac{L^{2} y}{\tau}} d s s^{-1-\frac{\zeta}{y}+\frac{\zeta^{\prime \prime}}{2 c}}(1+s)^{-\frac{7}{2}-\frac{\zeta^{\prime \prime}}{2 c}}
$$

We are now in a position to consider various limits. From (4.15) and 4.16) we get

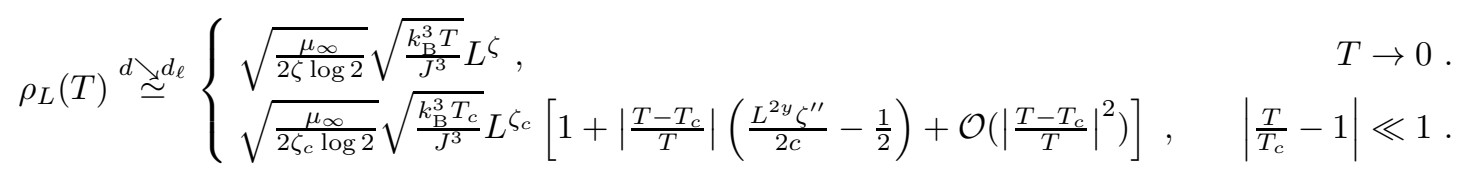

The expression (4.17 $\mathrm{b}$ ) shows that for large enough $L$ the density of zeros has a negative derivative at $T_{c}$, and must therefore have a maximum between $T=0$ and $T=T_{c}$. For smaller $L$, the derivative at $T_{c}$ is positive and the maximum of $\rho_{L}(T)$ must occur for $T>T_{c}$. The crossover value is

$$
L \stackrel{d}{\beth^{\beth}} d_{\ell} \exp \left(\frac{\log \left(1+\frac{c}{\zeta^{\prime \prime}}\right)}{2 y}\right)
$$

In the limit $d \searrow d_{\ell}$ at $L$ and $T$ fixed, we find

$$
\rho_{L}(T) \stackrel{d}{\beth^{\searrow}} d_{\ell} \sqrt{\frac{\mu_{\infty}}{2 \zeta \log 2}} \sqrt{\frac{k_{\mathrm{B}}^{3} T}{J^{3}}} L^{\zeta},
$$

valid in the whole interval of temperatures $0 \leqq T \leqq T_{c}$. The $\sqrt{T}$ behaviour at low temperatures was known for $d>d_{\ell}$ [3, 11, 12] and for $d<d_{\ell}$ [11, 12]. The present calculation shows that this behaviour persists when $d$ passes through $d_{\ell}$.

\section{Confluency of critical and zero-temperature phenomena near $d_{\ell}$}

A last comment on the confluency of critical and zero temperature properties within the geometric picture near $d_{\ell}$ is in place. Each sign reversal of a coupling $K_{R}^{L}$ on the temperature axis will typically be accompanied by a sign reversal of the correlation $\left\langle s_{0} s_{L}>\right.$ between two spins a distance $L$ apart in the region around $\boldsymbol{R}$. From (4.19) for the total number of sign reversals of $\left\langle s_{0} s_{L}\right\rangle$ between 0 and $T_{c}$

$$
\begin{aligned}
\mathcal{R}(L) & =\int_{0}^{T_{c}} d T \rho_{L}(T) \\
& \sim J^{-\frac{3}{2}} T_{c}^{\frac{3}{2}} L^{\zeta},
\end{aligned}
$$


valid near $d_{\ell}$. So, typically, as one passes from the ground state to the critical state there are no sign reversals of $s_{0}$ with respect to $s_{L}$ below a length scale $L_{\mathrm{gs}}$ given by

$$
L_{\mathrm{gs}} \sim\left(\frac{J}{k_{\mathrm{B}} T_{c}}\right)^{\frac{3}{2 \zeta}}
$$

Hence below this length scale, a protodroplet around a given point $\boldsymbol{R}$ at criticality coincides typically with the droplet at zero temperature in that region, even though an average over the whole system yields critical scaling and statistical invariance with length scale.

\section{SPIN-SPIN CORRELATIONS AND RESPONSE TO A MAGNETIC FIELD}

\section{A. Spin-spin correlation functions}

We wish to consider here spin-spin correlations at criticality. Instead of using the MKRG formalism as we did for the energy-energy correlations, we shall now make freely use of the interpretation in terms of thermally active surfaces developed in section $\amalg$ II B. For the following calculation we consider the thermally active surfaces as independent two-level systems, i.e., we neglect their interactions.

Let $\left\langle s_{i} s_{j}\right\rangle_{c}=<s_{i} s_{j}>-<s_{i}><s_{j}>$ be the connected correlation function between two spins a distance $L=2^{n}$ apart. In this section $i$ and $j$ denote spin sites and not, as before, bonds. By a "surface" enclosing a given spin at a given scale we mean in what follows the one with the lowest excitation energy of all possible such surfaces; it need not, and in general will not, be thermally active. With respect to the thermally active surfaces introduced above these surfaces are exactly what the droplets are to the thermally active droplets.

Let $k$ be the index denoting the surface of scale $2^{k}$ that encloses spin $i$ but not spin $j$, and let similarly $l$ be the index of the scale $2^{l}$ surface that encloses spin $j$ but not spin $i$. Furthermore let $m$ be the index of the scale $2^{m}$ surfaces that enclose both spins. We shall denote the excitation energies of these surfaces by $\epsilon_{k}, \epsilon_{l}$, and $\epsilon_{m}$, respectively.

The spin clusters enclosed by the surfaces introduced here can now be treated in the same way as the FH droplets in the ordered phase [3, 4 . For the spin-spin correlation it is sufficient to work in the approximation of independent surfaces; taking their interaction into account would only renormalise several of the constants but not lead to any new effects.

Upon treating the surfaces as independent two-level systems one finds

$$
<s_{i} s_{j}>_{c} \simeq s_{i}^{0} s_{j}^{0} \prod_{k=1}^{n-1} \tanh \frac{\beta \epsilon_{k}}{2} \prod_{l=1}^{n-1} \tanh \frac{\beta \epsilon_{l}}{2}\left[1-\prod_{m=n}^{\infty} \tanh ^{2} \frac{\beta \epsilon_{m}}{2}\right]
$$

where $s_{i}^{0} s_{j}^{0}$ is the relative orientation of $s_{i}$ and $s_{j}$ in the configuration with none of the surfaces excited. From this expression we obtain the averages

$$
\overline{<s_{i} s_{j}>^{2 q}} \simeq\left[\prod_{k=1}^{n-1} \overline{\tanh ^{2 q} \frac{\beta \epsilon_{k}}{2}}\right]^{2} \overline{\left[1-\prod_{m=n}^{\infty} \tanh ^{2} \frac{\beta \epsilon_{m}}{2}\right]^{2 q}} .
$$


At criticality, all the $\epsilon$ 's in the products are independently distributed random variables. Therefore the last factor in (5.2) equals unity and we find

$$
\overline{<s_{i} s_{j}>^{2 q}} \simeq e^{2 n \log \overline{\tanh ^{2 q} \frac{\beta \epsilon_{k}}{2}}} \sim L^{-q(d-2)-\eta_{c}^{(q)}}
$$

Obviously, we have in general $\eta_{c}^{(q)} \neq q \eta_{c}^{(1)} \equiv q \eta_{c}$. Hence, the $\eta_{c}^{(q)}$ are another series of independent critical exponents, i.e., we have also multifractal scaling of the spin-spin correlation function at criticality.

In [26], Sourlas considered the long-distance behaviour of correlation functions of critical spin glass using an effective field theory and the replica trick. For $d>6$, he found after averaging over the disorder that the different replicas are not coupled by relevant or marginal operators in the effective replicated Hamiltonian. It follows as in [27] that $\eta_{c}^{(q)}=0$ for $d>6$, i.e., the mean-field (Gaussian) behaviour. This is not the case in $d=6-\epsilon$ dimensions. An $\epsilon$-expansion around the effective field theory in $d=6$ leads to multifractality, i.e., $\eta_{c}^{(q)} \neq q \eta_{c}^{(1)}$, as found in this work by a very different approach.

\section{B. Response to a magnetic field}

\section{Introduction}

In this subsection, we will consider the magnetisation in a small uniform magnetic field $H$. Many of the results obtained in this section can be found scattered in previous work by several authors [1.225.23. McMillan [1], Bray and Moore [2,25], and Fisher and Huse [3, 1 ] analysed the effects of a magnetic field on the ordered phase of a spin glass by applying a variant of the argument used by Imry and Ma 28] to determine the stability of Ising ferromagnets to random fields. They find that the zero-field ordered states are destroyed beyond a length scale $\xi_{H}$ given by

$$
\xi_{H} \sim\left(\frac{\Upsilon(T)}{H \sqrt{q(T)}}\right)^{\frac{2}{d-2 y}},
$$

with $\Upsilon(T) \simeq J \xi_{-}^{-y}$ and $q(T) \simeq q_{0} \xi_{-}^{\beta}$. Equation (5.4) holds as long as the field $H$ is small enough so that $\xi_{H} \gtrsim \xi_{-}$. Here $\Upsilon(T)$ and $q(T)$ are the temperature-dependent stiffness coefficient and the EdwardsAnderson order parameter, respectively. The corresponding magnetisation $m(H)$ is calculated to be

$$
m(H)-\frac{q(T)}{y \Upsilon(T)} H \sim-\left(\frac{q^{\frac{1}{2}}(T) H}{\Upsilon(T)}\right)^{\frac{2 y}{d-2 y}} .
$$

The behaviour of $m(H)$ for $T=T_{c}$ can then be obtained on general scaling grounds [25, 1, 3 .

The picture developed in this paper now permits us to extend the Imry-Ma type argument to the low- $T$ critical point. The crucial property common to both the droplets of the ordered phase and the composite clusters at criticality is that they may reverse with respect to a fixed background. The effects of the magnetic field, in the ordered phase and at criticality, can therefore be summarised as follows: 
- The field aligns small composite clusters (thermally active or not) with a certain probability and larger ones with a larger probability and

- disrupts the fixed background by aligning practically all composite clusters from a certain length scale $\left(\sim \xi_{H}\right)$ on.

This allows us to present the effects of a magnetic field on the system in the ordered phase and at criticality in a unified way.

\section{A magnetic field at criticality}

\section{Temperatures below $T_{c}$}

We will first give the expressions for the correlation length $\xi_{H}$ in a field and for the magnetisation $m(H)$ for $T \leqq T_{c}$, valid irrespective of the relative size of the variables $t$ and $h$. In order to do that, we have to know the typical reversal free energy $\Delta F^{\ell}$ and the typical magnetic energy $E_{\mathrm{mag}}^{\ell}=H M^{\ell}$ of composite clusters, of linear size $\ell$, where $M^{\ell}$ denotes their total magnetisation. We recall that at length scales $\ell \gtrsim \xi_{-}$these composite clusters are the FH droplets. The reversal free energies $\Delta F^{\ell}$ are

$$
\Delta F^{\ell} \sim \begin{cases}J, & \ell \lesssim \xi_{-} . \\ \Upsilon(T) \ell^{y}, & \xi_{-} \lesssim \ell .\end{cases}
$$

In the ordered phase, the mean square value of the magnetisation is $q(T) \ell^{d}$ for large droplets. At criticality, we have to take into account the renormalisation of the order parameter

$$
q_{\ell}\left(T_{c}\right) \simeq q_{0} \ell^{-\frac{d-2+\eta_{c}}{2}} .
$$

Hence, for general $T$, the expression is

$$
M^{\ell} \sim \begin{cases}q_{0}^{\frac{1}{2}} \ell^{\frac{d+2-\eta_{c}}{4}}, & \ell \lesssim \xi_{-}, \\ q_{0}^{\frac{1}{2}} \xi_{-}^{-\frac{\beta}{\nu}} \ell^{\frac{d}{2}}, & \xi_{-} \lesssim \ell .\end{cases}
$$

From simulations (see 29] and references therein) and experiment [30] one can infer $\eta_{c} \approx-0.3$. In both cases $\eta_{c}$ is obtained by hyperscaling from the values of the critical susceptibility exponents.

Flipping a domain of scale $\ell$ will change the system's magnetisation by an amount of order $\ell^{\frac{d}{2}}$, the magnetisation of the domain going from $M^{\ell}$ to $-M^{\ell}$. Half of the composite clusters can lower their total free free energy by aligning with the field. Thus, the system breaks up into domains of typically the size of a correlation volume $\xi_{H}^{d}$. The correlation length $\xi_{H}$ is obtained by comparison of the reversal free energy and the magnetic energy as the solution of

$$
\Delta F^{\xi_{H}}=E_{\mathrm{mag}}^{\xi_{H}} .
$$

The typical fraction $f^{\ell}$ of domains of linear size $\ell \lesssim \xi_{H}$ that will reverse to align with the field is 


$$
f^{\ell}=\frac{E_{\mathrm{mag}}^{\ell}}{\Delta F^{\ell}}
$$

Finally, the total magnetisation $M(H)$ in a volume $\xi_{H}^{d}$ is given by the sum over all the domains, up to scale $\xi_{H}$, that align with the field:

$$
M(H)=H \int_{\sim 1}^{\xi_{H}} \frac{d \ell}{\ell} f^{\ell}\left(\frac{\xi_{H}}{\ell}\right)^{d} M^{\ell} .
$$

Hence the magnetisation (per spin) $m(H)$ is

$$
m(H)=H \int_{\sim 1}^{\xi_{H}} \frac{d \ell}{\ell} \frac{\left(M^{\ell}\right)^{2}}{\ell^{d} \Delta F^{\ell}} .
$$

Introducing (5.6) and (5.8) into (5.9), and solving for $\xi_{H}$, we get for the correlation length in a field near criticality:

$$
\xi_{H}=h^{-\frac{2 \nu}{\Delta}} \mathcal{F}\left(\frac{t}{h^{\frac{2}{\Delta}}}\right)
$$

where $\Delta=\nu\left(d+2-\eta_{c}\right) / 2$ is the gap exponent and hyperscaling is tacitly understood. Numerical values for $\Delta$ can be obtained with the help of the relation $\Delta=\beta+\gamma$ where $\gamma$ is the exponent describing the divergence of the nonlinear susceptibility. From Monte Carlo simulations and hightemperature series expansions one has estimated $\beta=0.6 \pm 0.1$ and $\gamma=2.95 \pm 0.25$ (see 29] and references therein). Analyses of experimental data give the same range of values for $\beta$ but favor a higher value of $\gamma=4.5 \pm 0.3$ [30]. The scaling (5.13) at criticality has been obtained by Fisher and Huse 3 previously [their equation (5.13)]. The scaling function $\mathcal{F}(x)$ satisfies

$$
\begin{aligned}
& \mathcal{F}(0)=\text { cst. }, \\
& \mathcal{F}(x) \quad \text { analytic for } x=0, \\
& \mathcal{F}(x) \stackrel{x \rightarrow \infty}{\sim}(-x)^{\frac{\Delta}{(d-2 y)}-1} .
\end{aligned}
$$

The magnetisation is obtained from (5.6), (5.8), (5.9) and (5.12):

$$
m(H)-\chi H=m_{\mathrm{sg}} \quad \text { with } \chi \sim \frac{2}{d-2+\eta_{c}} \frac{q_{0}}{J},
$$

and the singular part of the magnetisation $m_{\mathrm{sg}}$ verifies

$$
m_{\mathrm{sg}}(t, h) \sim-\frac{2 q_{0}^{\frac{1}{2}}}{d-2+\eta_{c}} h^{1+2 \frac{\beta}{\Delta}} \mathcal{M}\left(\frac{t}{h^{\frac{2}{\Delta}}}\right),
$$

with a scaling function $\mathcal{M}(x)$ such that $\mathcal{M}(0)$ is a positive constant, and

$$
\begin{array}{lr}
\mathcal{M}(x) & \text { analytic for } x=0, \\
\mathcal{M}(x) \stackrel{x \rightarrow-\infty}{\simeq} \frac{d-2+\eta_{c}}{2 y}(-x)^{\beta-\Delta \frac{y}{d-2 y}} .
\end{array}
$$


The limit $x \rightarrow-\infty$ is in agreement with the result obtained by Bray and Moore 2] [their equation (46)]. The linear term $\chi H$ exceeds the magnetisation $m(H)$ by the singular contribution $m_{\mathrm{sg}}(t, H)$, because, just as in the ordered phase [3], the low-field linear response of the domains with $L \gtrsim \xi_{H}$ has saturated.

In particular, we see from (5.16) and (5.17) that the exponent of $H$ in $m_{\mathrm{sg}}$ is $1+2 \frac{y}{d-2 y}$ and $1+2 \frac{\beta}{\Delta}$ for $x \rightarrow 0$ and $x \rightarrow-\infty$, respectively. Hence, the singular contribution is dominated by the linear response. However, both values of the exponent are smaller than 3 , so that the singular term dominates over the $H^{3}$ term in the magnetisation and causes the zero-field nonlinear susceptibility to be infinite. Furthermore, we see from (5.16) that the third derivative of the magnetisation diverges, for $H \rightarrow 0$, at the critical point as

$$
-\frac{d^{3} m}{d H^{3}} \simeq \frac{2 q_{0}^{\frac{1}{2}}}{d-2+\eta_{c}}\left(\frac{q_{0}^{\frac{1}{2}}}{J}\right)^{1+2 \frac{\beta}{\Delta}} H^{2\left(\frac{\beta}{\Delta}-1\right)} .
$$

For dimensions $d$ close above $d_{\ell}$ the following inequality holds:

$$
\frac{y}{d-2 y}<\frac{\beta}{\Delta} \Leftrightarrow d<\beta \frac{d-y}{\nu y} .
$$

The second one follows from the fact that, for $d \searrow d_{\ell}$, we have $\nu y \rightarrow \frac{1}{2}$ [3],$y \searrow 0$ and $\beta \sim y^{-\frac{1}{2}} \nearrow \infty$ (see [3]). It seems reasonable to assume that (5.19) is valid in general dimension $d>d_{\ell}$, so that the divergence for $H \rightarrow 0$ of $-\frac{d^{3} m}{d H^{3}}$ is stronger in the ordered phase than at criticality.

Summarising the above results for $H \rightarrow 0$ at $t$ fixed, we get:

$$
\begin{array}{cc}
T<T_{c}: \quad m(H)=a_{10} H-a_{s}(t) H^{1+\frac{2 y}{d-2 y}}+\ldots \\
\\
a_{s}(t) \simeq a_{s 0}|t|^{\beta-\frac{\Delta y}{d-2 y}} \\
T=T_{c}: \quad m(H)=a_{10} H-a_{s}^{c} H^{1+\frac{2 \beta}{\Delta}}+\ldots
\end{array}
$$

Here, $a_{10}, a_{s 0}$, and $a_{s}^{c}$ are amplitudes, and (5.19) implies that the exponent of $a_{s}(t)$ is positive.

\section{Temperatures above $T_{c}$}

Let $\xi_{+}$be the zero-field correlation length for $T>T_{c}$. Domains of linear size $\ell \lesssim \xi_{+}$behave critically, whereas much larger domains are paramagnetic. When $H$ is small, domains of size up to $\xi_{+}^{d}$ align partially and in order to obtain the magnetisation we have to integrate until scale $\xi_{+}$. When $H$ is larger, there is $\xi_{H}<\xi_{+}$, and the integration runs until $\xi_{H}$ as in equation (5.12). In the latter case, we get again the result (5.16), but with small positive argument of the scaling function. In the former case, we have

$$
m(H)=H \int_{\sim 1}^{\xi_{+}} \frac{d \ell}{\ell} \frac{\left(M^{\ell}\right)^{2}}{\ell^{d} \Delta F^{\ell}}
$$

so that 


$$
m(H)-\chi H \sim-\frac{2}{d-2+\eta_{c}} \frac{q_{0}}{J} t^{\beta} H, \quad \text { with } \quad \chi=\frac{2}{d-2+\eta_{c}} \frac{q_{0}}{J}
$$

the singular part of the magnetisation contributing a term $\sim t^{\beta}$ to the linear susceptibility.

We can write the magnetisation for $T \geqq T_{c}$, from (5.16) and (5.22), in a compact form:

$$
m(H)-\chi H \sim m_{\mathrm{sg}}
$$

with a constant contribution of the nonsingular part of the magnetisation to the linear susceptibility as in (5.22), whereas the singular part of the magnetisation behaves as

$$
m_{\mathrm{sg}}(t, h)=-\frac{2 q_{0}^{\frac{1}{2}}}{d-2+\eta_{c}} h^{1+2 \frac{\beta}{\Delta}} \mathcal{M}\left(\frac{t}{h^{\frac{2}{\Delta}}}\right)
$$

with $\mathcal{M}(0)$ a positive constant and the scaling function $\mathcal{M}(x)$ satisfies

$$
\begin{array}{ll}
\mathcal{M}(x) & \text { analytic for } x=0, \\
\mathcal{M}(x) \stackrel{x \rightarrow \infty}{\simeq} x^{\beta} .
\end{array}
$$

To study the limit $h \rightarrow 0$ at small positive $t$ of $(5.25)$, we write first

$$
\begin{aligned}
m_{\mathrm{sg}}(t, h) & \sim h^{1+2 \frac{\beta}{\Delta}} \mathcal{M}\left(\frac{t}{h \frac{2}{\Delta}}\right) \\
& =h^{3} t^{-\gamma} \hat{\mathcal{M}}\left(\frac{h^{2}}{t^{\Delta}}\right)
\end{aligned}
$$

where $\hat{\mathcal{M}}\left(\frac{h^{2}}{t^{\Delta}}\right) \equiv \frac{t^{\Delta}}{h^{2}} \mathcal{M}\left(\frac{t}{h^{\frac{2}{\Delta}}}\right)$. From $(5.25)$, we have

$$
\hat{\mathcal{M}}(u) \simeq u^{-1}, \quad u \rightarrow 0
$$

For $t>0$, the function $m_{\mathrm{sg}}$ should actually be nonsingular in $h$. Hence, with the assumption that $\hat{\mathcal{M}}$ is analytic in $h^{2}$, we get in summary for the magnetisation above the critical point:

$$
\begin{gathered}
T>T_{c}: \quad m(H)=a_{1}(t) H+a_{3}(t) H^{3}+\ldots \\
a_{1}(t) \simeq a_{10}-a_{1 \beta} t^{\beta}, \\
\\
a_{3}(t) \simeq a_{1 \gamma} t^{-\gamma}
\end{gathered}
$$

where $a_{10}, a_{1 \beta}$, and $a_{1 \gamma}$ are amplitudes. This shows that, for $t \searrow 0$, the linear susceptibility $\chi$ has a nondiverging singular contribution $\sim t^{\beta}$ and the well-known feature [25] that the nonlinear susceptibility diverges as $t^{-\gamma}$.

\section{A magnetic field at $d=d_{\ell}$}

We now turn to the special case of applying a small magnetic field $H$ at the lower critical dimension $d=d_{\ell}$. For $d \searrow d_{\ell}$, the contribution of the nonsingular part of the magnetisation to the linear 
susceptibility [see (5.15)] diverges, because $\eta_{c} \nearrow 2-d$. However, due to the simultaneous divergence of the singular part of the magnetisation [see (5.16), (5.24)] the linear susceptibility remains finite for $T>0$, because the leading contributions of the nonsingular and of the singular part cancel each other in the limit $d=d_{\ell}$, as previously observed by [1.25]. This gives rise to a logarithmic singularity of the magnetisation, for $H \rightarrow 0$ at $T=0$, which can be seen as follows. To obtain the expression of the magnetisation at $d=d_{\ell}$, we can reason as in the preceding subsection for $T \geqq T_{c}$, with here $T_{c}\left(d_{\ell}\right)=0$. At $T=0$, the reversal energy $\Delta F^{\ell}$ and the magnetisation $M^{\ell}$ of a cluster of linear size $\ell$ are

$$
\begin{aligned}
& \Delta F^{\ell} \sim J, \\
& M^{\ell} \sim q_{0}^{\frac{1}{2}} \ell^{\frac{d_{\ell}}{2}},
\end{aligned}
$$

for all length scales $\ell$, so that from (5.9)

$$
\xi_{H}=\left(\frac{J}{q_{0}^{\frac{1}{2}} H}\right)^{\frac{2}{d_{\ell}}} .
$$

However, for $T>0$, the renormalisation of the reversal free energy $\Delta F^{\ell}$ is nontrivial. Solving (4.2) for $y=0$ we obtain

$$
\Delta F^{\ell} \sim J \sqrt{1-c\left(\frac{k_{\mathrm{B}} T}{J}\right)^{2} \log \ell}
$$

as McMillan did previously [1]. The magnetisation, for $H \rightarrow 0$ at $T \geqq 0$, is readily calculated in the same way as above for $d>d_{\ell}$ and $T \geqq T_{c}$, the zero-field correlation length being $\xi_{+} \simeq e^{-\frac{1}{2 c f_{+}{ }^{2}}+\frac{J^{2}}{2 c\left(k_{\mathrm{B}} T\right)^{2}}}$ [see (4.8)]. One finds

$$
m(T, h)=\frac{q_{0}^{\frac{1}{2}} J}{k_{\mathrm{B}}} \frac{h}{T} \mathcal{M}_{d_{\ell}}\left(k_{\mathrm{B}} T \log h^{-1}\right)
$$

with a scaling function $\mathcal{M}_{d_{\ell}}$ that satisfies

$$
\begin{aligned}
& \mathcal{M}_{d_{\ell}}(x) \stackrel{x \rightarrow 0}{\simeq} x, \\
& \mathcal{M}_{d_{\ell}}(x) \stackrel{x \rightarrow \infty}{\simeq} 1 .
\end{aligned}
$$

Thus, in the limit $H \rightarrow 0$ at $T$ fixed, we obtain the Curie law as expected in the paramagnetic phase on general theoretical grounds [31].

\section{SOME COMMENTS ON CRITICAL DYNAMICS}

Random systems, like random field magnets and spin glasses, have far slower dynamics than conventional pure systems (see e.g. [32 34] and references therein). This is often attributed to fact that the competition between various types of interactions leads to free energy barriers of height $B^{L}$ that have to be crossed in thermally activated dynamical processes. If a static theory like the one of the 
preceding sections is to be extended to include dynamical phenomena, then additional assumptions concerning these barriers have to be made. It is generally assumed that a barrier typically grows with the length scale $L$ involved in the process as

$$
B^{L} \sim L^{\psi}
$$

with a barrier exponent $\psi>0$ and gives naturally rise to long time scales

$$
\tau^{L} \sim \exp \left(\frac{B^{L}}{k_{\mathrm{B}} T}\right)
$$

This type of scaling is called activated dynamic scaling. The activated processes can be either fluctuations in equilibrium or involve approach to equilibrium.

Generally, it has been agreed upon that this is indeed the mechanism valid at sufficiently large length scale in the spin glass ordered phase. However, it has remained unclear whether the critical dynamics in these systems should be described by activated or rather by conventional (power-law) dynamic scaling. Until now, most often critical dynamics has been scaled in a conventional manner, with anomalously large exponents, although thermally activated critical dynamic scaling with a small barrier exponent also appears to account reasonably well for experimental spin-glass behaviour at the critical point in three dimensions and does not seem to be incompatible with numerical simulations 34,35. Again, however, the scaling with either approach can be corrupted in $d=3$ due to a number of serious problems (see [30,29] and references therein for details).

The picture of this paper represents an extension of the droplet picture of the spin glass into the critical region. We argue in the following that it suggests that spin glass dynamics is activated also at the critical point, at least in dimensions close above $d_{\ell}$. We recall that in the FH theory the droplet walls minimise the free energy of a reversed spin domain. They are locally optimal and the fact that they dominate the physics of the ordered phase reflects the strong spatial inhomogeneity of thermal fluctuations in that phase. In particular, to reverse a droplet of scale $L$, the domain wall must pass through regions of high free-energy cost which are larger than the excitation free energy of the completed droplet. Therefore FH argue that droplet reversal is a thermally activated process with barriers $B^{L}$ behaving as in (6.1) and growing at least as fast with length scale as the free energy difference between initial and reversed state. Therefore its barrier exponent satisfies

$$
y(d) \leqq \psi(d)
$$

in dimensions $d$ above $d_{\ell}[3$.

The same reasoning can be applied to the protodroplets of low-dimensional spin glass at criticality. But the lower bound $0 \leqq \psi_{c}$ for the scaling of the barriers at criticality, obtained from comparison with the scaling of the free energy, is trivial. Indeed, $\psi_{c}=0$ would mean conventional scaling. We now give an argument that points towards $\psi_{c}(d)>0$ in an interval of dimensions extending upward from $d_{\ell}$. Dynamics near zero temperature in two dimensions has been argued on the basis of numerical simulations and experiments to be thermally activated. Numerical work [36] points to $\psi(2) \approx 1$ (but see also [34] and references therein) and experiments give $\psi(2) /|y(2)|=1.6 \pm 0.2$ and $\psi(2) /|y(2)|=0.9 \pm 0.1$ for $40 \AA$ [37] and $\lesssim 20 \AA$ [38] thick spin glass films respectively. This would mean $\psi(d)>0$, and, since the barrier exponent is expected to increase with dimension, also $\psi\left(d_{\ell}\right)>0$. 
Hence by continuity $\psi_{c}(d)>0$ in an interval of dimensions upward from $d_{\ell}$. In these dimensions the barrier heights scale at criticality like

$$
B^{L}\left(T_{c}\right) \sim J L^{\psi_{c}}, \quad 0<\psi_{c} .
$$

Hence, the characteristic reversal time $\tau^{L}$ of a composite cluster at a large scale $L$ at criticality is exponentially length scale dependent:

$$
\tau^{L} \sim \tau_{0} \exp \frac{J L^{\psi_{c}}}{k_{\mathrm{B}} T_{c}}
$$

Such a behaviour implies [3] that ac correlation functions should scale, in the critical region, as functions of $\frac{\log \left(\omega \tau_{0}\right)}{\xi_{-}^{\psi_{c}}}$ and that

$$
\chi_{3}(3 \omega ; \omega) \sim\left|\log \frac{\gamma}{\psi_{c \nu}}\left(\omega \tau_{0}\right)\right|
$$

i.e., the cubic nonlinear susceptibility diverges only logarithmically with vanishing frequency at criticality.

\section{CONCLUSION}

We have analysed the critical state of the low-dimensional Ising spin glass, in particular in the limit of the dimension $d$ approaching the lower critical dimensionality $d_{\ell}$.

The best existing approximate theory for the low-dimensional spin glass is the scaling or droplet theory developed since the middle 1980's by McMillan, Bray and Moore, Fisher and Huse, and others

近 4 . The building blocks of this theory, in the language of Fisher and Huse, are the low free energy excitations, called droplets, from the spin glass ordered state. Droplets are reversed regions of one phase embedded in the opposite phase. Their excitation energy has a random value on an energy scale that increases with their size. The droplet wall, like in ferromagnetism, has a width of the order of the correlation length. Consequently droplets exist only in the ordered phase and the droplet theory in its original formulation says nothing about criticality.

We show in this work that the FH theory has a natural extension into the critical region, that is, to length scales less than the correlation length. This extension can be presented as a heuristic expansion around the dimension $d_{\ell}$. Its main idea is summarised as follows.

In a critical state very close to $d_{\ell}$ most bonds are frozen, but there is a very dilute set of surfaces (of width zero) that enclose clusters of spins able to thermally reverse with respect to their environment. The density $p$ of these surfaces is the expansion parameter; it is proportional to the critical temperature $T_{c}$. Because of scale invariance there is the same density $p$ of thermally active surfaces at all length scales. Since $p$ is small but nonzero, interactions between these surfaces (through common surface sections) should be taken into account. Therefore, in the low-dimensional critical state the thermally active degrees of freedom are organised in finite disjoint sets of interacting thermally active surfaces. These sets have a distribution of sizes that ranges to infinity. Each set has a self-similar structure on scales from its own size down to the lattice cutoff. Such a set of interacting surfaces is appropriately 
called a protodroplet. In the off-critical limit, that is, for length scales beyond $\xi_{-}$, one of the surfaces participating in a protodroplet will dominate in size so that all the others may be considered as thermally decorating this large one and building up its wall: the protodroplet has then become a droplet.

This picture of the critical state explains the phenomenon of critical chaos [11,12. It has enabled us to discuss the effects of a magnetic field in a unified way, using an Imry-Ma type argument in the ordered phase and at criticality. Finally, we have briefly argued, on the basis of additional hypotheses, that in low enough dimensions the critical dynamics is thermally activated.

The picture of this paper has the practical advantage of making definite statements about spin glass properties on length scales below the correlation length (in contrast to the Fisher and Huse droplet picture, which is valid on scales above the correlation length). Some of our predictions should be easily accessible in simulations of the Edwards-Anderson model. One may of course attempt to determine the existence and properties of the thermally active surfaces (they exist from the scale of the lattice distance up), but also more conventionally verify our predictions for various spatial correlation functions.

The following three remarks about possible future sinulations are in no way meant to be exhaustive.

1. About thermally active cluster surfaces. A quantity that measures the degree of thermal activity of the surface element perpendicular to a bond between two neighbouring spins $s_{i}$ and $s_{i^{\prime}}$ is $\left|<s_{i} s_{i^{\prime}}\right\rangle \mid$. If this quantity is zero, the bond is strongly thermally active; if it is close to unity the bond is nearly frozen. Its spatial (or disorder) average is equal to $1-\mathcal{O}\left(k_{B} T_{c} / J\right)$. Conceivably a chart of all nearest neighbour $\left|<s_{i} s_{i^{\prime}}\right\rangle \mid$ could be converted at the end of the simulation into a map of all thermally active surfaces.

2. Dimension dependence. As the dimension $d$ moves up from $d_{\ell}$, the critical temperature $T_{c}$ moves away from 0 and thermally active cluster surfaces become gradually harder to distinguish, since almost all bonds will acquire some degree of thermal activity. Also, the thermally active surfaces, even if they can be distinguished, may, from some dimension on, form infinite percolating structures and the theory of our paper is not directly applicable. In dimension $d=2$ and at sufficiently low temperature, the thermally active cluster surfaces exist on length scales less than the correlation length $\xi$, but the correlation functions are not multifractal; beyond $\xi$ the surfaces form an infinitely connected structure. Therefore $\mathrm{d}=3$ is the ideal dimension for testing our predictions.

3. Analysis in terms of correlation functions. This is the more conventional approach. The theory predicts power laws at distances less than the correlation length (but of course sufficiently large with respect to the lattice cutoff; how much larger we do not know). The powers are in general different for different moments of a given correlation function. We extract from the preceding sections what seem to us the most important ones.

The disorder averaged energy-energy correlation function $\overline{\left\langle\Delta e_{i} \Delta e_{j}\right\rangle}$, equation $(2.28)$, should behave as (see equations $(2.29)$ and 2.34$)$ ):

$$
\overline{<\Delta e_{i} \Delta e_{j}>} \sim|i-j|^{2 y_{c}-2 d} \quad\left(d>d_{\ell}, \quad|i-j|<\xi_{-}\right) .
$$

However, the theory also says that $\left\langle\Delta e_{i} \Delta e_{j}\right\rangle$ has fluctuations larger than its average value. Its variance (see equations (2.30) and (2.37)) is 


$$
\overline{\left\langle\Delta e_{i} \Delta e_{j}\right\rangle^{2}}-\overline{\left\langle\Delta e_{i} \Delta e_{j}\right\rangle^{2}} \sim|i-j|^{4 \zeta_{c}-2 d} .
$$

(The Migdal-Kadanoff values for $d=3$ are $y_{c} \approx 0.36$ and $\zeta_{c} \approx 0.57$ 11, 12, 18.) We are not aware of any direct determination yet of the exponent $\zeta_{c}$ in the Edwards-Anderson model. The corresponding zero temperature exponent $\zeta$ has in $d=3$ the Migdal-Kadanoff value $\zeta \approx 0.75$ [11, 12, 18]; the difference $\zeta-\zeta_{c}$, just as the difference $y_{c}-y$, measures the distance to the lower critical dimension. The higher moments $\overline{\left\langle\Delta e_{i} \Delta e_{j}\right\rangle^{2 q}}$ and $\overline{\left\langle\Delta e_{i} \Delta e_{j}\right\rangle^{2 q-1}}$ decay with powers of $|i-j|$ that involve a hierarchy of exponents $\zeta_{c}(q)$, see equation (2.38).

A quantity that should be measurable with relatively good precision is the fourth energy

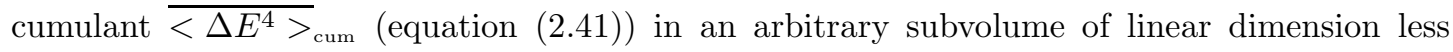
than the correlation length. As $V$ becomes large,

$$
\frac{\overline{\left\langle\Delta E^{4}\right\rangle_{\text {cum }}}}{V} V \rightarrow \infty\left\{\begin{array}{rll}
V^{4 \frac{\zeta_{c}}{d}-1} & \text { if } & 4 \zeta_{c} / d>1 \\
\text { cst. - (terms nonanalytic in } V) & \text { if } & 4 \zeta_{c} / d<1
\end{array}\right.
$$

The MKRG values indicate that in $d=3$ the second scenario will be realised, since numerically $4 \zeta_{c} / d-1=4 \cdot 0.57 / 3-1=-0.24$. However, we cannot be sure of the sign of this quantity in $d=3$. At $d_{\ell}$, MKRG gives for the same quantity the value of approximately $4 \cdot 0.75 / 2.5-1=0.2$. Hence the fourth energy cumulant in $d=3$ provides a - possibly rather sensitive - way of determining $\zeta_{c}$.

Acknowledgements. One of us (M.J.T.) wishes to thank David Huse for many valuable discussions related to the subject.

[1] W.L. McMillan, J. Phys. C 17 (1984) 3179.

[2] A.J. Bray and M.A. Moore, Scaling theory of the ordered phase of Ising spin glasses, in: Heidelberg Colloquium on Glassy Dynamics, J.L. van Hemmen and I. Morgenstern (eds), Springer-Verlag, Heidelberg (1986).

[3] D.S. Fisher and D.A. Huse, Phys. Rev. Lett. 56 (1986) 1601; D.S. Fisher and D.A. Huse, Phys. Rev. B 38 (1988) 386 .

[4] D.S. Fisher and D.A. Huse, Phys. Rev. B 36 (1987) 8937; D.S. Fisher and D.A. Huse, J. Phys. A 20 (1987) L997; D.S. Fisher and D.A. Huse, J. Phys. A 20 (1987) L1005; D.S. Fisher and D.A. Huse, Phys. Rev. B 38 (1988) 373.

[5] A.J. Bray and M.A. Moore, Phys. Rev. B 34 (1986) 6561, and references therein.

[6] S.F. Edwards et P.W. Anderson, J. Phys. F 5 (1975) 965. 
[7] A.A. Migdal, Zh. Eksp. Teor. Fiz. 69 (1975) 1457 [Sov. Phys. JETP 42 (1975) 743]; L.P. Kadanoff, Ann. Phys. (New York) 91 (1975) 226.

[8] A.P. Young and R.B. Stinchcombe, J. Phys. C 9 (1976) 4419.

[9] B.W. Southern and A.P. Young, J. Phys. C 10 (1977) 2179.

[10] P.W. Anderson and C.M. Pond, Phys. Rev. Lett. 40 (1978) 903.

[11] M. Nifle and H.J. Hilhorst, Phys. Rev. Lett. 68 (1992) 2992.

[12] M. Ney-Nifle and H.J. Hilhorst, Physica A 193 (1993) 48.

[13] J.R. Banavar and A.J. Bray, Phys. Rev. B 35 (1987) 8888.

[14] A.J. Bray and M.A. Moore, J. Phys. C 17 (1984) L463; W.L. McMillan, Phys. Rev. B 30 (1984) 476; Phys. Rev. B 31 (1985) 340.

[15] W.L. McMillan, Phys. Rev. B 29 (1984) 4026; A.J. Bray and M.A. Moore, Phys. Rev. B 31 (1985) 631.

[16] R.G. Caflisch, J.R. Banavar, and M. Cieplak, J. Phys. C 18 (1985) L991.

[17] R.R.P. Singh and M.E. Fisher, J. Appl. Phys. 63 (1988) 3994; M.E. Fisher and R.R.P. Singh, in: Disorder in Physical Systems, G. Grimmett and D.J.A. Welsh (eds), Oxford University Press, Oxford, 1990.

[18] M.J. Thill and H.J. Hilhorst, unpublished.

[19] P. Collet and J.-P. Eckmann, Commun. Math. Phys. 93 (1984) 379.

[20] For some properties of multifractal exponents and applications of multifractal scaling with other systems see e.g. B. Duplantier and A. W. W. Ludwig, Phys. Rev. Lett. 66 (1991) 247 and references therein.

[21] A.J. Bray and M.A. Moore, Phys. Rev. Lett. 58 (1987) 57.

[22] M. Cieplak, A. Maritan, and J.R. Banavar, J. Phys. A 27 (1994) L765.

[23] M. Cieplak, A. Maritan, and J.R. Banavar, Phys. Rev. Lett. 72 (1994) 2320.

[24] D.A. Huse, Phys. Rev. B 43 (1991) 8673.

[25] A.J. Bray and M.A. Moore, J. Phys. C 17 (1984), L613.

[26] N. Sourlas, Europhys. Lett. 3 (1987) 1007.

[27] G. Parisi, Phys. Lett. B 136 (1984) 392.

[28] Y. Imry and S.-K. Ma, Phys. Rev. Lett. 35 (1975) 1399.

[29] L. Klein, J. Adler, A. Aharony, A.B. Harris, Y. Meir, Phys. Rev. B 43 (1991) 11249.

[30] S. Geschwind, D.A. Huse, and G.E. Devlin, Phys. Rev. B 41 (1990) 4854; Phys. Rev. B 41 (1990) 2650.

[31] A.C.D. van Enter and J.L. van Hemmen, Phys. Rev. A 29 (1984) 355.

[32] D.S. Fisher, J. Appl. Phys. 61 (1987) 3672; D.A. Huse and D.S. Fisher, Phys. Rev. B 35 (1987) 6841.

[33] D.H. Ryan (ed.), Recent Progress in Random Magnets, World Scientific, Singapore (1992); J.A. Mydosh, Spin glasses: an experimental introduction, Taylor \& Francis, London (1993). 
[34] H. Rieger, Monte Carlo Studies of Ising Spin Glasses and Random Field Systems, in: Annual Reviews of Computational Physics II , D. Stauffer (ed.), World Scientific, Singapore (1995).

[35] D.A. Huse, Phys. Rev. B 43 (1991) 8673.

[36] A.P. Young, Phys. Rev. Lett. 50 (1983) 917; W. Kinzel and K. Binder, Phys. Rev. B 29 (1984) 1300; D.S. Fisher and D.A. Huse, Phys. Rev. B 36 (1987) 8937.

[37] L. Sandlund, P. Granberg, L. Lundgren, P. Nordblad, P. Svedlindh, J.A. Cowen, and G.G. Kenning, Phys. Rev. B 40 (1989) 869; L. Hoines, R. Stubi, R. Loloee, J.A. Cowen, and J. Bass, Phys. Rev. Lett. 66 (1991) 1224.

[38] J. Mattsson, private communication. 